Article

\title{
stu Black Holes Unveiled
}

\author{
Stefano Bellucci ${ }^{1}$, Sergio Ferrara ${ }^{2,1,3}$, Alessio Marrani ${ }^{4,5,1 \star}$ and Armen Yeranyan ${ }^{1,6}$ \\ ${ }^{1}$ INFN - Laboratori Nazionali di Frascati, Via Enrico Fermi 40, I-00044 Frascati, Italy \\ ${ }^{2}$ Physics Department,Theory Unit, CERN, CH 1211, Geneva 23, Switzerland \\ ${ }^{3}$ Miller Institute for Basic Research in Science, University of California, Berkeley, CA94720, USA \\ ${ }^{4}$ Museo Storico della Fisica e Centro Studi e Ricerche "Enrico Fermi", I-00184 Roma, Italy \\ ${ }^{5}$ Stanford Institute for Theoretical Physics, Stanford University, Stanford CA, 94305-4060, USA \\ ${ }^{6}$ Department of Physics, Yerevan State University, Alex Manoogian St., 1, Yerevan, 375025, Armenia \\ E-mails: bellucci@lnf.infn.it; sergio.ferrara@cern.ch; marrani@lnf.infn.it; ayeran@lnf.infn.it
}

* Author to whom correspondence should be addressed.

Received: 5 October 2008 / Accepted: 13 October 2008 / Published: 17 October 2008

\begin{abstract}
The general solutions of the radial attractor flow equations for extremal black holes, both for non-BPS with non-vanishing central charge $Z$ and for $Z=0$, are obtained for the so-called stu model, the minimal rank-3 $\mathcal{N}=2$ symmetric supergravity in $d=4$ space-time dimensions.

Comparisons with previous results, as well as the fake supergravity (first order) formalism and an analysis of the BPS bound all along the non-BPS attractor flows and of the marginal stability of corresponding $D$-brane configurations, are given.
\end{abstract}

Keywords: black holes; attractors; supergravity.

\section{Introduction}

The physics of black holes (BHs) [1]-[6] has received much attention in the last years. This is also due to the issue of the Attractor Mechanism[7]-[10], a general phenomenon which occurs in extremal BHs coupled to Maxwell and scalar fields, as it is the case in supersymmetric theories of gravity [11][74] (for further developments, see also e.g. [75]-[78]).

Supergravity [79] can be obtained as the low-energy (small curvature expansion) limit of superstrings [80]- [83] or M-theory [84-86]; in such a framework, a certain number of $U$ (1) gauge fields and moduli 
fields are coupled to the Einstein-Hilbert action. This is especially the case for theories in $d=4$ spacetime dimensions, and having $\mathcal{N} \geqslant 2$ supercharges, where $4 \mathcal{N}$ is the number of supersymmetries. A popular example is the compactification of Type II superstring theory on a Calabi-Yau threefold $(\mathcal{N}=$ 2 ) or on a six-torus $(\mathcal{N}=8)$. The fermionic sector of these theories contains a certain number of spin $1 / 2$ fermions and $\mathcal{N}$ spin $3 / 2$ Rarita-Schwinger fields, named gravitinos (the gauge fields of local supersymmetry). The vanishing of the supersymmetric variation of the gravitinos determines whether or not a certain number of supersymmetries (BPS property) is preserved by the BH background.

In this situation, asymptotically flat charged $\mathrm{BH}$ solutions, within a static and spherically symmetric Ansatz, can be regarded as a generalization of the famous Schwarzschild BH. However, the presence of additional quantum numbers (such as charges and scalar hair) make their properties change drastically, and new phenomena appear. A novel important feature of electrically (and/or magnetically) charged BHs [87] as well as rotating ones [88] is a somewhat unconventional thermodynamical property named extremality [6, 92, 93]. Extremal BHs are possibly stable gravitational objects with finite entropy but vanishing temperature, in which case the contribution to the gravitational energy entirely comes from the electromagnetic (charges) and rotational [27] (angular momentum/spin) attributes. Extremality also means that the inner (Cauchy) and outer (event) horizons do coincide, thus implying vanishing surface gravity (for a recent review see e.g. [70], and Refs. therein).

In the regime of extremality a particular relation among entropy, charges and spin holds, yielding that the Arnowitt-Deser-Misner (ADM) gravitational mass [89-91] is not an independent quantity. Stationary and spherically symmetric BHs in $d=4$ space-time dimensions and in an environment of scalar fields (typically described by a non-linear sigma model) have scalar hair (scalar charges), corresponding to the values of the scalars at (asymptotically flat) spatial infinity. These values may continuously vary, being an arbitrary point in the moduli space of the theory or, in a more geometrical language, a point in the target manifold of the scalar non-linear Lagrangian [7, 94]. Nevertheless, the BH entropy, as given by the Bekenstein-Hawking entropy-area formula [95], is also in this case independent on the scalar charges ("no scalar hair") and it only depends on the asymptotic (generally dyonic) BH charges.

This apparent puzzle can be resolved thanks to the aforementioned Attractor Mechanism, a fascinating phenomenon that combines extremal BHs, dynamical systems, algebraic geometry and number theory [2]. It was firstly discovered in the context of supergravity; in a few words, in constructing extremal dyonic BHs of $\mathcal{N}=2, d=4$ ungauged supergravity coupled to vector and hypermultiplets (with no $d=4$ scalar potential), two phenomena occur: the hyperscalars can take arbitrary constant values, while the radial evolution of the vector multiplets' scalars is described by a dynamical system [8, 9]. Under some mild assumptions, the scalar trajectory flows to a "fixed point", located at the BH event horizon, in the target (moduli) space. The "fixed point" (i.e. a point of vanishing phase velocity) represents the system in equilibrium, and it is the analogue of an attractor in the dynamical flow of dissipative systems. In approaching such an attractor, the orbits lose practically all memory of initial conditions (i.e. of the "scalar hair"), even though the dynamics is fully deterministic. The scalars at the BH horizon turn out to depend only on the dyonic (asymptotic) BH charges.

All extremal static, spherically symmetric and asymptotically flat BHs in $d=4$ have a BertottiRobinson [96] $A d S_{2} \times S^{2}$ near-horizon geometry, with vanishing scalar curvature and conformally flat; in particular, the radius of $A d S_{2}$ coincides with the radius of $S^{2}$, and it is proportional to the (square root 
of the) BH entropy (in turn proportional, through the Bekenstein-Hawking formula [95], to the area of the event horizon). Non-BPS (i.e. non-supersymmetric) (see e.g. [10, 26, 41, 43, 57, 59, 60]) extremal BHs exist as well, and they also exhibit an attractor behavior.

A particularly remarkable $\mathcal{N}=2, d=4$ ungauged supergravity is the so-called stu model, which exhibits the noteworthy triality symmetry [15, 39, 41, 50, 97-100]. It has been recently shown to be relevant for the analogy between pure states of multipartite entanglement of qubits in quantum information theory and extremal stringy BHs [3].

The 3 complex scalars coming from the 3 Abelian vector multiplets coupled to the supergravity one span the rank-3, completely factorized special Kähler manifold $\frac{G}{H}=\left(\frac{S U(1,1)}{U(1)}\right)^{3}$, with $\operatorname{dim}_{\mathbb{C}}=3$,

$$
G=(S U(1,1))^{3} \sim(S O(2,1))^{3} \sim(S L(2, \mathbb{R}))^{3} \sim(S p(2, \mathbb{R}))^{3}
$$

being the $d=4 U$-duality group, and $H=(U(1))^{3} \sim(S O(2))^{3}$ its maximal compact subgroup. Such a space is nothing but the element $n=2$ of the sequence of reducible homogeneous symmetric special Kähler manifolds $\frac{S U(1,1)}{U(1)} \otimes \frac{S O(2, n)}{S O(2) \otimes S O(n)}$ (see e.g. [26] and Refs. therein). It is here worth pointing out that, with a slight abuse of language, we refer to $U$-duality group as to the continuous version, valid for large values of charges, of the string duality group introduced by Hull and Townsend [101].

The $s t u$ model has 2 non-BPS $Z \neq 0$ flat directions, spanning the moduli space $S O(1,1) \times S O(1,1)$ (i.e. the scalar manifold of the stu model in $d=5$ ), but no non-BPS $Z=0$ massless Hessian modes at all [43] (see also [41] and [40]). In other words, the $6 \times 6$ Hessian matrix of the effective BH potential at its non-BPS $Z \neq 0$ critical points has 4 strictly positive and 2 vanishing eigenvalues (these latter correspond to massless Hessian modes), whereas at its non-BPS $Z=0$ critical points all the eigenvalue are strictly positive. After [10], $\frac{1}{2}$-BPS critical points of $V_{B H}$ in $\mathcal{N}=2, d=4$ supergravity are all stable, and thus they determine attractors in a strict sense. It is here worth pointing out that the $d=6$ uplift of the stu model is $(1,0)$ supergravity coupled to $n_{T}=1$ tensor multiplet. The $\mathrm{BH}$ charge orbits supporting the various classes of non-degenerate attractors have been studied in [26] (see also [50]).

Concerning its stringy origins, the $s t u$ model can be interpreted e.g. as the low-energy limit of Type $I I A$ superstrings compactified on a six-torus $T^{6}$ factorized as $T^{2} \times T^{2} \times T^{2}$. The $D 0-D 2-D 4-D 6$ branes wrapping the various $T^{2}$ s determine the 4 magnetic and 4 electric $\mathrm{BH}$ charges.

Remarkably, the stu model is a sector of all $\mathcal{N}>2, d=4$ supergravities, as well as of all $\mathcal{N}=2$, $d=4$ supergravities based on homogeneous (both symmetric [97, 113-115] and non-symmetric - see e.g. $[116,117]$-) scalar manifolds based on cubic geometries. Thus, stu model captures the essential features of extremal BHs in all such theories (see e.g. the $s t u$ interpretation of $\mathcal{N}=8, d=4$ attractors [24], and the observations in [59]).

Recently, the stu model has been object of detailed investigation concerning the integration of the equations of motion of the scalars in the background of a given BH charge configuration, in particular of those ones which support non-supersymmetric flows:

- In [28] the non-BPS $Z \neq 0$ attractor flow was investigated for the first time. The $\frac{1}{2}$-BPS attractor flow solution was previously studied in [105]-[109], and its most general form is known to be obtained simply by replacing the $\mathrm{BH}$ charges with the corresponding, symplectic covariant harmonic 
functions in the the most general horizon, critical solution, obtained in [99, 100]. In [28] such a feature has been shown to hold also for non-BPS $Z \neq 0$ attractor flow, for the $D 2-D 6$ (electric) and $D 0-D 2-D 4-D 6$ configurations, for particular cases without B-fields.

- In [51] the D0 - D4 (magnetic) system was considered, and new exact non-BPS $Z \neq 0$ attractor flow solutions were derived, with non-vanishing dynamical axion. Furthermore, the result of [28] was proved to be non-general. Indeed, within the $D 0-D 4$ (magnetic) configuration (dual to the electric one, studied in [28]), it was pointed out that non-BPS $Z \neq 0$ attractor flow differ from the $\frac{1}{2}$-BPS one, because the most general non-BPS $Z \neq 0$ solution cannot be obtained by replacing the $\mathrm{BH}$ charges with the corresponding harmonic functions in the most general horizon critical solution, as instead it holds for the supersymmetric case [105]-[109], and actually also for the non-BPS $Z=0$ case (see Sect. 4.). This is actually due to the presence of non-trivial so-called $B$-fields in the non-BPS $Z \neq 0$ attractor flow, as well as to the presence of flat directions (spanning a related moduli space) all along such a flow.

- In [59] the stu model was further studied in the D0-D4 (magnetic) as well as in the D0-D6 configurations, by fully exploiting the contribution of the B-fields, and (as also done in [28]) performing the relevant $U$-duality transformations in order to relate different $\mathrm{BH}$ charge configurations supporting the same attractor flow (i.e. belonging to the same $\mathrm{BH}$ charge orbit of $U$-duality [26]). The ADM mass $M_{A D M}$ of the extremal BH was computed in the $\frac{1}{2}$-BPS and in the non-BPS $Z \neq 0$ cases, and the marginal stability [111] of the corresponding physical states was studied, founding that the marginal bound [111] was saturated in the non-supersymmetric case, contrarily to the BPS case. The difference between the squared non-BPS $Z \neq 0$ ADM mass $M_{A D M, n o n-B P S, Z \neq 0}^{2}$ and $|Z|^{2}$ was computed at the radial infinity along the non-BPS $Z \neq 0$ attractor flow, showing that the BPS bound [112] actually holds also at the infinity, even if dependent on the asymptotical values of the scalars (see Eq. (4.8) of [59]). Moreover, in such a paper the two non-BPS $Z \neq 0$ flat directions of the stu model $[41,43]$ were shown to hold also along the whole corresponding attractor flow, as mentioned above.

- The analysis of [59] was further developed in [62], in which the non-BPS $Z \neq 0$ equations of motion of the scalars were solved for the D2-D6 (electric) and D0 - D2 - D4 supporting BH charge configurations.

The present paper is devoted to a detailed, complete study of the attractor flow equations of the stu model, whose fundamental facts are summarized in Sect. 2.. All the classes of non-degenerate (i.e. with non-vanishing classical Bekenstein-Hawking [95] BH entropy) attractor flow solutions are determined, in their most general form (with all B-fields switched on). The main results of our investigation are listed below:

- As mentioned above, the $\frac{1}{2}$-BPS attractor flow solution is known since [105]-[109], and it is reviewed in Sect. 3.. In Sect. 4. the non-BPS $Z=0$ attractor flow solution, untreated so far, is determined for the most general supporting BH charge configuration, and its relation to the supersymmetric flow, both at and away from the event horizon radius $r_{H}$, is established, consistently with the results of [50]. 
- Sect. 5. is devoted to the study of the non-BPS $Z \neq 0$ attractor flow solution in full generality. By using suitable $U$-duality transformations (Subsect. 5.1.), and starting from the $D 0-D 6$ configuration (Subsect. 5.2.), the non-BPS $Z \neq 0$ attractor flow supported by the most general $D 0-D 2-D 4-D 6$ configuration (with all charges switched on) is explicitly derived in Subsect. 5.3.. This completes and generalizes the analyses and the results of [28], [51], [59] and [62]. The above mentioned finding of [59] is confirmed in such a general framework: the moduli space $(S O(1,1))^{2}$, known to exist at the non-BPS $Z \neq 0$ critical points of $V_{B H}[41,43]$, is found to be present all along the non-BPS attractor flow, i.e. for every $r \geqslant r_{H}$.

- In Sect. 6. a detailed analysis of particular configurations, namely D0 - D4 (magnetic, Subsect. 6.1.), its dual D2-D6 (electric, Subsect. 6.2.), and D0-D2-D4 (Subsect. 6.3.), is performed.

- The so-called first order (fake supergravity) formalism, introduced in [118], has been recently developed in [37] and [42] in order to describe $d=4$ extremal BHs; in general, it is based on a suitably defined real, scalar-dependent, fake superpotential $\mathcal{W}$. It is worth pointing out that the first order formalism, as (re)formulated in [37] and [42] for $d=4$ extremal BHs, automatically selects the solutions which do not blow up at the BH event horizon. In other words, the (covariant) scalar charges $\Sigma_{i}$ built in terms of the fake superpotential $\mathcal{W}$ (see Eq. (20) further below) satisfy by construction all the conditions in order for the Attractor Mechanism to hold.

It should be here recalled that for extremal BHs the solution converging at the $\mathrm{BH}$ event horizon $\left(r \rightarrow r_{H}^{+}\right)$does not depend on the initial, asymptotical values of the scalar fields. See $e . g$. discussions in [33] and [44].

In the framework of stu model, we explicitly build up $\mathcal{W}$ in the non-trivial cases represented by the non-BPS attractor flows. For the non-BPS $Z=0$ attractor flow (Sect. 4.) a manifestly $H$ invariant $\mathcal{W}$ is determined, in three different (but equivalent) "polarizations". Furthermore, the difference between the squared non-BPS $Z=0$ fake superpotential $\mathcal{W}_{n o n-B P S, Z=0}^{2}$ and $|Z|^{2}$ is computed along the non-BPS $Z=0$ attractor flow, and the BPS bound [112] is found to hold all along the attractor flow (i.e. not only on the BH event horizon $r=r_{H}$, but $\forall r \geqslant r_{H}$ ). On the other hand, for non-BPS $Z \neq 0$ attractor flow (Subsects. 5.2. and 5.3.) the fake superpotential is manifestly not $H$-invariant.

As it will be commented in Sect. 7., this is not inconsistent with the treatment of [37] and [42]. Indeed, the fake superpotential is not unique within the same attractor flow, the various equivalent superpotentials being related through a (possibly scalar-dependent) $R$-matrix satisfying the conditions (2.28) and (2.29) of [37] (see in general Subsect. 2.2 of [37]). Moreover, in Sect. 6. the difference between the squared non-BPS $Z \neq 0$ fake superpotential $\mathcal{W}_{n o n-B P S, Z \neq 0}^{2}$ and $|Z|^{2}$ is computed along the non-BPS $Z \neq 0$ attractor flow in the magnetic (Subsect. 6.1.), electric (Subsect. 6.2.) and $D 0-D 2-D 4$ (Subsect. 6.3.) charge configurations. Analogously to the non-BPS $Z=0$ case, the BPS bound [112] is found to hold all along the attractor flow (i.e. not only on the $\mathrm{BH}$ event horizon $r=r_{H}$, but $\forall r \geqslant r_{H}$ ). In particular, for the magnetic charge configuration at radial infinity the result given by Eq. (4.8) of [59] is recovered. We are grateful to E. G. Gimon for a clarifying discussion about the definition of "gap" above the BPS bound as given in [59]. 
- Within the first order (fake supergravity) formalism, for all attractor flows we compute the covariant scalar charges as well as the ADM mass, studying the issue of marginal stability [111]. We thus complete the analysis and the results of [51], [59] and [62]. As expected due to the strict similarity to the $\frac{1}{2}$-BPS attractor flow (Sect. 3.), also in the non-BPS $Z=0$ case the marginal bound is not saturated (Sect. 4.), as instead we confirm to hold in general in the (non-BPS $Z \neq 0$-supporting branch of the) $D 0-D 2-D 4-D 6$ configuration (Subsect. 5.3.).

- Final remarks, comments, and outlook for further developments are given in the concluding Sect. 7..

\section{Basics of the $s t u$ Model}

We here recall some basic facts of the above mentioned stu model [15, 28, 39-41, 43, 50, 97-100], fixing our notations and conventions. The three complex moduli of the model are defined as

$$
z^{1} \equiv x^{1}-i y^{1} \equiv s, z^{2} \equiv x^{2}-i y^{2} \equiv t, z^{3} \equiv x^{3}-i y^{3} \equiv u
$$

with $y^{i} \in \mathbb{R}_{0}^{+}$[122] (see also the treatment of [47]). In special coordinates (see e.g. [119] and Refs. therein) the prepotential determining the relevant special Kähler geometry simply reads

$$
f=s t u \text {. }
$$

Due to the aforementioned triality symmetry among $s, t$ and $u$, the expressions of all the relevant geometric quantities acquire quite elegant form. Some formulæ can be found e.g. in the treatments of $[28,50,59,99,100]$; for completeness, below we list the expressions of the Kähler potential, contravariant metric tensor, non-vanishing components of the Christoffel symbols of the second kind and of the $C$-tensor, holomorphic central charge (also named superpotential) and BH effective potential $(i=1,2,3$ throughout):

$$
\begin{aligned}
& K=-\ln [-i(s-\bar{s})(t-\bar{t})(u-\bar{u})] \Rightarrow \exp (-K)=8 y^{1} y^{2} y^{3} ; \\
& g^{i \bar{j}}=-\operatorname{diag}\left((s-\bar{s})^{2},(t-\bar{t})^{2},(u-\bar{u})^{2}\right) ; \\
& \Gamma_{11}^{1}=-2(s-\bar{s})^{-1}, \Gamma_{22}^{2}=-2(t-\bar{t})^{-1}, \Gamma_{33}^{3}=-2(u-\bar{u})^{-1} ; \\
& C_{s t u}=\frac{i}{(s-\bar{s})(t-\bar{t})(u-\bar{u})}=\exp (K) ; \\
& W(s, t, u)=q_{0}+q_{1} s+q_{2} t+q_{3} u+p^{0} s t u-p^{1} t u-p^{2} s u-p^{3} s t ; \\
& V_{B H}=\exp (K) \cdot\left[|W(s, t, u)|^{2}+|W(\bar{s}, t, u)|^{2}+|W(s, \bar{t}, u)|^{2}+|W(s, t, \bar{u})|^{2}\right] .
\end{aligned}
$$


Thus, the (covariantly holomorphic) central charge function for the stu model reads (see e.g. [119] and Refs. therein)

$$
\begin{aligned}
Z(s, t, u, \bar{s}, \bar{t}, \bar{u}) & \equiv e^{K / 2} W(s, t, u)= \\
& =\frac{1}{\sqrt{-i(s-\bar{s})(t-\bar{t})(u-\bar{u})}}\left(q_{0}+q_{1} s+q_{2} t+q_{3} u+p^{0} s t u-p^{1} t u-p^{2} s u-p^{3} s t\right) .
\end{aligned}
$$

The definition of the $\mathrm{BH}$ charges $p^{\Lambda}$ (magnetic) and $q_{\Lambda}$ (electric) $(\Lambda=0,1,2,3$ throughout), the effective 1-dim. (quasi-)geodesic Lagrangian of the stu model, and the corresponding Eqs. of motion for the scalars can be found in Subsects. 2.2 and 2.3, as well as in appendix A (treating the case D0 - D4 in detail), of [59].

Through the Bekenstein-Hawking entropy-area formula [95], the entropy of an extremal $\mathrm{BH}$ in the stu model in the Einsteinian approximation reads as follows:

$$
S_{B H}=\frac{A_{H}}{4}=\left.\pi V_{B H}\right|_{\partial V_{B H}=0}=\pi \sqrt{\left|\mathcal{I}_{4}(\Gamma)\right|}
$$

where the $\left(2 n_{V}+2\right) \times 1$ vector of $\mathrm{BH}$ charges

$$
\Gamma \equiv\left(p^{\Lambda}, q_{\Lambda}\right)
$$

was introduced, $n_{V}$ denoting the number of Abelian vector multiplets coupled to the supergravity one (in the case under consideration $n_{V}=3$ ). Furthermore, $\mathcal{I}_{4}(\Gamma)$ denotes the unique invariant of the trifundamental representation $(2,2,2)$ of the $U$-duality group $G$, reading as follows (see e.g. Eq. (4.10) and Sect. 5 of [50], and Refs. therein):

$$
\mathcal{I}_{4}(\Gamma)=-\left(p^{\Lambda} q_{\Lambda}\right)^{2}+4 \sum_{i<j} p^{i} q_{i} p^{j} q_{j}-4 p^{0} q_{1} q_{2} q_{3}+4 q_{0} p^{1} p^{2} p^{3}=-\operatorname{Det}(\Psi),
$$

where $\operatorname{Det}(\Psi)$ is the so-called Cayley's hyperdeterminant [3].

In the next three Sections we will discuss the explicit solutions of the equations of motion of the scalars $s, t$ and $u$ in the dyonic background of an extremal $\mathrm{BH}$ of the stu model, also named Attractor Flow Equations. We will consider only non-degenerate attractor flows, i.e. those flows determining a regular, non-vanishing area of the horizon in the Einsteinian approximation.

As mentioned above, 3 classes of non-degenerate attractor flows exist in the stu model:

- $\frac{1}{2}$-BPS (Sect. 3.);

- non-BPS $Z=0$ (Sect. 4.);

- non-BPS $Z \neq 0$ (Sects. 5.) and (6.).

\section{The Most General $\frac{1}{2}$-BPS Attractor Flow}

The explicit expression of the attractor flow solution supported by the most general $\frac{1}{2}$-BPS BH charge configuration in $\mathcal{N}=2, d=4$ ungauged supergravity coupled to $n_{V}$ Abelian vector multiplets (and 
exhibiting a unique $U$-invariant $\mathcal{I}_{4}$ ) is known after [105]-[109] (as well as the third of Refs. [111]):

$$
\begin{aligned}
\exp \left[-4 U_{\frac{1}{2}-B P S}(\tau)\right] & =\mathcal{I}_{4}(\mathcal{H}(\tau)) \\
z_{\frac{1}{2}-B P S}^{i}(\tau) & =\frac{H^{i}(\tau)+i \partial_{H_{i}} \mathcal{I}_{4}^{1 / 2}(\mathcal{H}(\tau))}{H^{0}(\tau)+i \partial_{H_{0}} \mathcal{I}_{4}^{1 / 2}(\mathcal{H}(\tau))}
\end{aligned}
$$

where $\partial_{H_{i}} \equiv \frac{\partial}{\partial H_{i}}$, and the $\left(2 n_{V}+2\right) \times 1(=8 \times 1$ in the model under consideration) symplectic vector

$$
\mathcal{H}(\tau) \equiv\left(H^{\Lambda}(\tau), H_{\Lambda}(\tau)\right)
$$

was introduced, where $H^{\Lambda}(\tau)$ and $H_{\Lambda}(\tau)$ are harmonic functions defined as follows $\left(\tau \equiv\left(r_{H}-r\right)^{-1} \in\right.$ $\mathbb{R}^{-}$):

$$
\begin{aligned}
& H^{\Lambda}(\tau) \equiv p_{\infty}^{\Lambda}+p^{\Lambda} \tau ; \\
& H_{\Lambda}(\tau)=q_{\Lambda, \infty}+q_{\Lambda} \tau,
\end{aligned}
$$

such that $\mathcal{H}(\tau)$ can be formally rewritten as

$$
\mathcal{H}(\tau)=\Gamma_{\infty}+\Gamma \tau
$$

The asymptotical constants $\Gamma_{\infty}$ must satisfy the following integrability conditions:

$$
\mathcal{I}_{4}\left(\Gamma_{\infty}\right)=1,\left\langle\Gamma, \Gamma_{\infty}\right\rangle=0
$$

where $\langle\cdot, \cdot\rangle$ is the scalar product defined by the $\left(2 n_{V}+2\right) \times\left(2 n_{V}+2\right)$ symplectic metric. Under such conditions, the flow (9) is the most general solution of the so-called $\frac{1}{2}$-BPS stabilization Eqs. (see e.g. the recent treatment of [28]):

$$
\mathcal{H}^{T}(\tau)=2 e^{K(z(\tau), \bar{z}(\tau))} \operatorname{Im}\left[W(z(\tau), \mathcal{H}(\tau))\left(\begin{array}{c}
\bar{X}^{\Lambda}(\bar{z}(\tau)) \\
\bar{F}_{\Lambda}(\bar{z}(\tau))
\end{array}\right)\right],
$$

obtained from the $\frac{1}{2}$-BPS Attractor Eqs. (see e.g. the treatment in [23], and Refs. therein)

$$
\Gamma^{T}=2 e^{K(z, \bar{z})} \operatorname{Im}\left[W(z, \Gamma)\left(\begin{array}{c}
\bar{X}^{\Lambda}(\bar{z}) \\
\bar{F}_{\Lambda}(\bar{z})
\end{array}\right)\right]
$$

by simply replacing $\Gamma$ with $\mathcal{H}(\tau)$ (see e.g. [106] and Refs. therein). Consistently, Eq. (15) is the near-horizon $(\tau \rightarrow-\infty)$ limit of Eq. (14).

Moreover, the $\mathrm{BH}$ charge configurations supporting the $\frac{1}{2}$-BPS attractors at the BH event horizon satisfy the following constraints, defining the $\frac{1}{2}$-BPS orbit (see Appendix II of [26])

$$
\mathcal{O}_{\frac{1}{2}-B P S}=\frac{(S U(1,1))^{3}}{(U(1))^{2}}
$$


of the tri-fundamental representation $(\mathbf{2}, \mathbf{2}, \mathbf{2})$ of the $U$-duality group $(S U(1,1))^{3}[26,50]$ :

$$
\begin{aligned}
& \mathcal{I}_{4}(\Gamma)>0 ; \\
& p^{2} p^{3}-p^{0} q_{1} \gtrless 0 ; \\
& p^{1} p^{3}-p^{0} q_{2} \gtrless 0 ; \\
& p^{1} p^{2}-p^{0} q_{3} \gtrless 0 .
\end{aligned}
$$

Correspondingly, $\mathcal{H}(\tau)$ is constrained as follows along the $\frac{1}{2}$-BPS attractor flow $\left(\forall \tau \in \mathbb{R}^{-}\right)$:

$$
\begin{aligned}
& \mathcal{I}_{4}(\mathcal{H}(\tau))>0 ; \\
& H^{2}(\tau) H^{3}(\tau)-H^{0}(\tau) H_{1}(\tau) \gtrless 0 ; \\
& H^{1}(\tau) H^{3}(\tau)-H^{0}(\tau) H_{2}(\tau) \gtrless 0 ; \\
& H^{1}(\tau) H^{2}(\tau)-H^{0}(\tau) H_{3}(\tau) \gtrless 0 .
\end{aligned}
$$

In the near-horizon limit $\tau \rightarrow-\infty$, Eq. (9) yields the purely charge-dependent, critical expressions of the scalars at the $\mathrm{BH}$ event horizon, e.g. given by Eq. (3.1) of [59]. In the same limit, the constraints (18) consistently yield the constraints (17).

Consistently with the analysis of [47], the general $\frac{1}{2}$-BPS attractor flow solution (9) of the stu model can be axion-free only for the configurations $D 0-D 6, D 0-D 4$ (magnetic) and D2-D6 (electric).

As found in [121] and observed also in [59], an immediate consequence of Eq. (9) is that $\Gamma_{\infty}$ satisfies the $\frac{1}{2}$-BPS Attractor Eqs. [106]. This determines a sort of "Attractor Mechanism at spatial infinity”, mapping the 6 real moduli $\left(x^{1}, x^{2}, x^{3}, y^{1}, y^{2}, y^{3}\right)$ into the 8 real constants $\left(p_{\infty}^{1}, p_{\infty}^{2}, p_{\infty}^{3}, q_{1, \infty}, q_{2, \infty}, q_{3, \infty}\right)$, arranged as $\Gamma_{\infty}$ and constrained by the 2 real conditions (13).

As noticed in [59], the absence of flat directions in the $\frac{1}{2}$-BPS attractor flow (which is a general feature of $\mathcal{N}=2, d=4$ ungauged supergravity coupled to Abelian vector multiplets, at least as far as the metric of the scalar manifold is strictly positive definite $\forall \tau \in \mathbb{R}^{-}$[10]) is crucial for the validity of the expression (9).

Now, by exploiting the first order formalism [118] for $d=4$ extremal BHs [37, 42] (see also [70] and [74]), one can compute the relevant BH parameters of the $\frac{1}{2}$-BPS attractor flow of the stu model starting from the expression of the $\frac{1}{2}$-BPS fake superpotential $\mathcal{W}_{\frac{1}{2}-B P S}$. For instance, the ADM mass and covariant scalar charges respectively read (see e.g. the treatments in [70] and [74]):

$$
\begin{aligned}
M_{A D M}\left(z_{\infty}, \bar{z}_{\infty}, \Gamma\right) & =\mathcal{W}\left(z_{\infty}, \bar{z}_{\infty}, \Gamma\right) \equiv \lim _{\tau \rightarrow 0^{-}} \mathcal{W}(z(\tau), \bar{z}(\tau), \Gamma) \\
\Sigma_{i}\left(z_{\infty}, \bar{z}_{\infty}, \Gamma\right) & =\left(\partial_{i} \mathcal{W}\right)\left(z_{\infty}, \bar{z}_{\infty}, \Gamma\right) \equiv \lim _{\tau \rightarrow 0^{-}}\left(\partial_{i} \mathcal{W}\right)(z(\tau), \bar{z}(\tau), \Gamma),
\end{aligned}
$$

where the subscript " $\infty$ " denotes the evaluation at the moduli at spatial infinity $\left(r \rightarrow \infty \Leftrightarrow \tau \rightarrow 0^{-}\right.$). Notice that Eq. (19) provides, within the considered first order formalism, an alternative (eventually 
simpler) formula for the computation of $M_{A D M}$, with respect to the general definition in terms of the warp factor $U$ (see e.g. [10]):

$$
M_{A D M}=\lim _{\tau \rightarrow 0^{-}} \frac{d U(\tau)}{d \tau} .
$$

Recalling that for all $\mathcal{N}=2, d=4$ ungauged supergravities it holds that $\mathcal{W}_{\frac{1}{2}-B P S}=|Z|$, Eqs. (5) and (19) yield the following expressions of the ADM mass of the $\frac{1}{2}$-BPS attractor flow of the stu model:

$$
\begin{gathered}
M_{A D M, \frac{1}{2}-B P S}\left(z_{\infty}, \bar{z}_{\infty}, \Gamma\right) \equiv \lim _{\tau \rightarrow 0^{-}}|Z|(z(\tau), \bar{z}(\tau), \Gamma)= \\
=\frac{\left|q_{0}+q_{1} s_{\infty}+q_{2} t_{\infty}+q_{3} u_{\infty}+p^{0} s_{\infty} t_{\infty} u_{\infty}-p^{1} t_{\infty} u_{\infty}-p^{2} s_{\infty} u_{\infty}-p^{3} s_{\infty} t_{\infty}\right|}{\sqrt{-i\left(s_{\infty}-\bar{s}_{\infty}\right)\left(t_{\infty}-\bar{t}_{\infty}\right)\left(u_{\infty}-\bar{u}_{\infty}\right)}} .
\end{gathered}
$$

Eq. (22) yields that the marginal bound [111] is not saturated by $\frac{1}{2}$-BPS states, because $M_{A D M, \frac{1}{2}-B P S}$ is not equal to the sum of the ADM masses of four D6-branes with appropriate fluxes (for further detail, see the discussion in [59]).

Concerning the (covariant) scalar charges of the $\frac{1}{2}$-BPS attractor flow of the stu model, they can be straightforwardly computed by using Eqs. (5) and (20):

$$
\begin{gathered}
\sum_{s, \frac{1}{2}-B P S}\left(z_{\infty}, \bar{z}_{\infty}, \Gamma\right)= \\
=\lim _{\tau \rightarrow 0^{-}} \frac{e^{K / 2}}{2}\left[\left(\partial_{s} K\right)|W|+\left(\partial_{s} W\right) \sqrt{\frac{W}{W}}\right](z(\tau), \bar{z}(\tau), \Gamma)= \\
=\frac{1}{2 \sqrt{-i\left(s_{\infty}-\bar{s}_{\infty}\right)\left(t_{\infty}-\bar{t}_{\infty}\right)\left(u_{\infty}-\bar{u}_{\infty}\right)}} \cdot \\
\cdot\left[\frac{\left|q_{0}+q_{1} s_{\infty}+q_{2} t_{\infty}+q_{3} u_{\infty}+p^{0} s_{\infty} t_{\infty} u_{\infty}-p^{1} t_{\infty} u_{\infty}-p^{2} s_{\infty} u_{\infty}-p^{3} s_{\infty} t_{\infty}\right|}{\left(s_{\infty}-\bar{s}_{\infty}\right)}+\right. \\
\left.+\sqrt{\frac{q_{0}+q_{1} \bar{s}_{\infty}+q_{2} \bar{t}_{\infty}+q_{3} \bar{u}_{\infty}+p^{0} \bar{s}_{\infty} \bar{t}_{\infty} \bar{u}_{\infty}-p^{1} \bar{t}_{\infty} \bar{u}_{\infty}-p^{2} \bar{s}_{\infty} \bar{u}_{\infty}-p^{3} \bar{s}_{\infty} \bar{t}_{\infty}}{q_{0}+q_{1} s_{\infty}+q_{2} t_{\infty}+q_{3} u_{\infty}+p^{0} s_{\infty} t_{\infty} u_{\infty}-p^{1} t_{\infty} u_{\infty}-p^{2} s_{\infty} u_{\infty}-p^{3} s_{\infty} t_{\infty}}}\right] .
\end{gathered}
$$




$$
\begin{aligned}
& \Sigma_{t, \frac{1}{2}-B P S}\left(z_{\infty}, \bar{z}_{\infty}, \Gamma\right)= \\
& =\lim _{\tau \rightarrow 0^{-}} \frac{e^{K / 2}}{2}\left[\left(\partial_{t} K\right)|W|+\left(\partial_{t} W\right) \sqrt{\frac{\bar{W}}{W}}\right](z(\tau), \bar{z}(\tau), \Gamma)= \\
& =\frac{1}{2 \sqrt{-i\left(s_{\infty}-\bar{s}_{\infty}\right)\left(t_{\infty}-\bar{t}_{\infty}\right)\left(u_{\infty}-\bar{u}_{\infty}\right)}} \text {. } \\
& \cdot\left[\frac{\left|q_{0}+q_{1} s_{\infty}+q_{2} t_{\infty}+q_{3} u_{\infty}+p^{0} s_{\infty} t_{\infty} u_{\infty}-p^{1} t_{\infty} u_{\infty}-p^{2} s_{\infty} u_{\infty}-p^{3} s_{\infty} t_{\infty}\right|}{\left(t_{\infty}-\bar{t}_{\infty}\right)}+\right. \\
& +\left(q_{2}+p^{0} s_{\infty} u_{\infty}-p^{1} u_{\infty}-p^{3} s_{\infty}\right) \cdot \\
& \left.\cdot \sqrt{\frac{q_{0}+q_{1} \bar{s}_{\infty}+q_{2} \bar{t}_{\infty}+q_{3} \bar{u}_{\infty}+p^{0} \bar{s}_{\infty} \bar{t}_{\infty} \bar{u}_{\infty}-p^{1} \bar{t}_{\infty} \bar{u}_{\infty}-p^{2} \bar{s}_{\infty} \bar{u}_{\infty}-p^{3} \bar{s}_{\infty} \bar{t}_{\infty}}{q_{0}+q_{1} s_{\infty}+q_{2} t_{\infty}+q_{3} u_{\infty}+p^{0} s_{\infty} t_{\infty} u_{\infty}-p^{1} t_{\infty} u_{\infty}-p^{2} s_{\infty} u_{\infty}-p^{3} s_{\infty} t_{\infty}}}\right] . \\
& \Sigma_{u, \frac{1}{2}-B P S}\left(z_{\infty}, \bar{z}_{\infty}, \Gamma\right)= \\
& =\lim _{\tau \rightarrow 0^{-}} \frac{e^{K / 2}}{2}\left[\left(\partial_{u} K\right)|W|+\left(\partial_{u} W\right) \sqrt{\frac{\bar{W}}{W}}\right](z(\tau), \bar{z}(\tau), \Gamma)= \\
& =\frac{1}{2 \sqrt{-i\left(s_{\infty}-\bar{s}_{\infty}\right)\left(t_{\infty}-\bar{t}_{\infty}\right)\left(u_{\infty}-\bar{u}_{\infty}\right)}} \text {. } \\
& \cdot\left[\frac{\left|q_{0}+q_{1} s_{\infty}+q_{2} t_{\infty}+q_{3} u_{\infty}+p^{0} s_{\infty} t_{\infty} u_{\infty}-p^{1} t_{\infty} u_{\infty}-p^{2} s_{\infty} u_{\infty}-p^{3} s_{\infty} t_{\infty}\right|}{\left(u_{\infty}-\bar{u}_{\infty}\right)}+\right. \\
& +\left(q_{3}+p^{0} s_{\infty} t_{\infty}-p^{1} t_{\infty}-p^{2} s_{\infty}\right) \cdot \\
& \left.\cdot \sqrt{\frac{q_{0}+q_{1} \bar{s}_{\infty}+q_{2} \bar{t}_{\infty}+q_{3} \bar{u}_{\infty}+p^{0} \bar{s}_{\infty} \bar{t}_{\infty} \bar{u}_{\infty}-p^{1} \bar{t}_{\infty} \bar{u}_{\infty}-p^{2} \bar{s}_{\infty} \bar{u}_{\infty}-p^{3} \bar{s}_{\infty} \bar{t}_{\infty}}{q_{0}+q_{1} s_{\infty}+q_{2} t_{\infty}+q_{3} u_{\infty}+p^{0} s_{\infty} t_{\infty} u_{\infty}-p^{1} t_{\infty} u_{\infty}-p^{2} s_{\infty} u_{\infty}-p^{3} s_{\infty} t_{\infty}}}\right] .
\end{aligned}
$$

\section{The Most General Non-BPS $Z=0$ Attractor Flow}

Let us now investigate the non-BPS $Z=0$ case.

As shortly noticed in [59], in spite of the fact that this attractor flow is non-supersymmetric, it has many common features with the supersymmetric $\left(\frac{1}{2}-\mathrm{BPS}\right)$ case.

As yielded by the analysis of [50], the non-BPS $Z=0$ horizon attractor solutions can be obtained from $\frac{1}{2}$-BPS ones simply by changing the signs of any two imaginary parts of the moduli (dilatons) and consistently imposing specific constraints on BH charges. For example, one can choose (without any loss of generality, due to triality symmetry) to flip the dilatons as follows:

$$
y^{1} \rightarrow y^{1}, y^{2} \rightarrow-y^{2}, y^{3} \rightarrow-y^{3} .
$$


This yields the following constraints on the BH charge configurations supporting the non-BPS $Z=0$ attractors at the $\mathrm{BH}$ event horizon $(\tau \rightarrow-\infty)$ [50]:

$$
\begin{aligned}
& \mathcal{I}_{4}(\Gamma)>0 ; \\
& p^{2} p^{3}-p^{0} q_{1} \lessgtr 0 ; \\
& p^{1} p^{3}-p^{0} q_{2} \gtrless 0 ; \\
& p^{1} p^{2}-p^{0} q_{3} \gtrless 0 .
\end{aligned}
$$

The constraints (27) defines the non-BPS $Z=0$ orbit of the tri-fundamental representation $(2,2,2)$ of the $U$-duality group $(S U(1,1))^{3}$ (see Appendix II of [26])

$$
\mathcal{O}_{n o n-B P S, Z=0}=\frac{(S U(1,1))^{3}}{(U(1))^{2}} .
$$

Notice that such an orbit shares the same coset expression of $\mathcal{O}_{\frac{1}{2}-B P S}$ given by Eq. (16). However, they do not coincide, but instead they are two separated branches of a disconnected manifold, classified by the local value of the function $\operatorname{sgn}\left(|Z|^{2}-\left|D_{s} Z\right|^{2}\right.$ ) (see Appendix II of [26]; $D_{s} Z$ is defined below Eq. (38)).

The same holds all along the attractor flow, i.e. $\forall \tau \in \mathbb{R}^{-}$. Indeed, the most general non-BPS $Z=0$ attractor flow can be obtained by taking the most general $\frac{1}{2}$-BPS attractor flow, and flipping any two out of the three dilatons. Thus, by taking Eq. (9) and flipping the dilatons as given by Eq. (26), one achieves the following result:

$$
\begin{aligned}
\exp \left[-4 U_{\text {non-BPS,Z=0 }}(\tau)\right] & =\mathcal{I}_{4}(\mathcal{H}(\tau)) ; \\
z_{\text {non-BPS,Z=0}}^{1}(\tau) & =\frac{H^{\Lambda}(\tau) H_{\Lambda}(\tau)-2 H^{1}(\tau) H_{1}(\tau)-i \mathcal{I}_{4}^{1 / 2}(\mathcal{H}(\tau))}{2\left[H^{2}(\tau) H^{3}(\tau)-H^{0}(\tau) H_{1}(\tau)\right]}=z_{\frac{1}{2}-B P S}^{1}(\tau) ; \\
z_{\text {non-BPS,Z=0}}^{2}(\tau) & =\frac{H^{\Lambda}(\tau) H_{\Lambda}(\tau)-2 H^{2}(\tau) H_{2}(\tau)+i \mathcal{I}_{4}^{1 / 2}(\mathcal{H}(\tau))}{2\left[H^{1}(\tau) H^{3}(\tau)-H^{0}(\tau) H_{2}(\tau)\right]}=\overline{z_{\frac{1}{2}-B P S}^{2}}(\tau) ; \\
z_{\text {non-BPS,Z=0}}^{3}(\tau) & =\frac{H^{\Lambda}(\tau) H_{\Lambda}(\tau)-2 H^{3}(\tau) H_{3}(\tau)+i \mathcal{I}_{4}^{1 / 2}(\mathcal{H}(\tau))}{2\left[H^{2}(\tau) H^{1}(\tau)-H^{0}(\tau) H_{3}(\tau)\right]}=\overline{z_{\frac{1}{2}-B P S}^{3}}(\tau) .
\end{aligned}
$$

This is the most general expression of the non-BPS $Z=0$ attractor flow, in the "polarization" given by Eq. (26), which, due to the underlying triality symmetry of the stu model, does not imply any loss of generality.

Consistently with the constraints $(27), \mathcal{H}(\tau)$ is constrained as follows along the non-BPS $Z=0$ 
attractor flow $\left(\forall \tau \in \mathbb{R}^{-}\right)$:

$$
\begin{aligned}
& \mathcal{I}_{4}(\mathcal{H}(\tau))>0 ; \\
& H^{2}(\tau) H^{3}(\tau)-H^{0}(\tau) H_{1}(\tau) \lessgtr 0 ; \\
& H^{1}(\tau) H^{3}(\tau)-H^{0}(\tau) H_{2}(\tau) \gtrless 0 ; \\
& H^{1}(\tau) H^{2}(\tau)-H^{0}(\tau) H_{3}(\tau) \gtrless 0 .
\end{aligned}
$$

In the near-horizon limit $\tau \rightarrow-\infty$, Eq. (29) yields the purely charge-dependent, critical expressions of the scalars at the BH event horizon, given by Eq. (4.9) of [50]. In the same limit, the constraints (30) consistently yield the contraints (27). The integrability conditions (13) clearly hold also in this case.

Consistently with the analysis of [47], the general non-BPS $Z=0$ attractor flow solution (29) of the stu model can be axion-free only for the configurations $D 0-D 6, D 0-D 4$ (magnetic) and D2 - D6 (electric).

A consequence of Eq. (29) is that $\Gamma_{\infty}$ satisfies the non-BPS $Z=0$ Attractor Eqs. (see e.g. [23] and [32]). Analogously to what happens for the $\frac{1}{2}$-BPS attractor flow, this determines a sort of "Attractor Mechanism at spatial infinity”.

Analogously to what happens in the $\frac{1}{2}$-BPS case, the absence of $f$ lat directions in the non-BPS $Z=0$ attractor flow (which is not a general feature of $\mathcal{N}=2, d=4$ ungauged supergravity coupled to Abelian vector multiplets, but however holds for the stu model $[40,43])$ is crucial for the validity of the expression (29).

By exploiting the strict relation with the $\frac{1}{2}$-BPS attractor flow, one can also determine the explicit expression of the fake superpotential $\mathcal{W}_{\text {non-BPS,Z=0 }}$ for the non-BPS $Z=0$ attractor flow. Considering the absolute value of the $\mathcal{N}=2, d=4$ central charge function $Z$ given by Eq. (5) and flipping two dilatons out of three in the "polarization" given by Eq. (26), one obtains the following non-BPS $Z=0$ fake superpotential (notice that $K$, as given by the first Eq. of (4), is invariant under such a flipping):

$$
\begin{aligned}
\mathcal{W}_{\text {non-BPS,Z=0,s }} & =e^{K / 2}\left|q_{0}+q_{1} s+q_{2} \bar{t}+q_{3} \bar{u}+p^{0} s \bar{t} \bar{u}-p^{1} \bar{t} \bar{u}-p^{2} s \bar{u}-p^{3} s \bar{t}\right|= \\
& =|Z(s, \bar{t}, \bar{u})|=\mathcal{W}_{\frac{1}{2}-B P S}(s, \bar{t}, \bar{u})
\end{aligned}
$$

where the subscript " $s$ " denotes the modulus untouched by the considered flipping of dilatons; in the last step we used that aforementioned fact that for all $\mathcal{N}=2, d=4$ ungauged supergravities it holds that $\mathcal{W}_{\frac{1}{2}-B P S}=|Z|$.

Clearly, the flipping (26) is not the only possible one. By triality symmetry, two other equivalent flippings exist, namely

$$
\begin{aligned}
& y^{1} \rightarrow-y^{1}, y^{2} \rightarrow y^{2}, y^{3} \rightarrow-y^{3} ; \\
& y^{1} \rightarrow-y^{1}, y^{2} \rightarrow-y^{2}, y^{3} \rightarrow y^{3},
\end{aligned}
$$

obtained by cyclic permutations from (26). Such equivalent flippings respectively determine the following (respectively " $t$-polarized" and "u-polarized”) non-BPS $Z=0$ fake superpotentials, completely 
equivalent to the "s-polarized" one given by Eq. (31):

$$
\begin{aligned}
& \mathcal{W}_{\text {non-BPS,Z=0,t}}=e^{K / 2}\left|q_{0}+q_{1} \bar{s}+q_{2} t+q_{3} \bar{u}+p^{0} \bar{s} t \bar{u}-p^{1} t \bar{u}-p^{2} \overline{s u}-p^{3} \bar{s} t\right|= \\
& =|Z(\bar{s}, t, \bar{u})|=\mathcal{W}_{\frac{1}{2}-B P S}(\bar{s}, t, \bar{u}) \text {; } \\
& \mathcal{W}_{\text {non-BPS,Z=0,u}}=e^{K / 2}\left|q_{0}+q_{1} \bar{s}+q_{2} \bar{t}+q_{3} u+p^{0} \bar{s} \bar{t} u-p^{1} \bar{t} u-p^{2} \bar{s} u-p^{3} \bar{s} \bar{t}\right|= \\
& =|Z(\bar{s}, \bar{t}, u)|=\mathcal{W}_{\frac{1}{2}-B P S}(\bar{s}, \bar{t}, u) \text {. }
\end{aligned}
$$

It can be shown by straightforward computations that the real, moduli- and charge- dependent functions given by Eqs. (31), (34) and (35) do satisfy all the conditions defining a first order fake superpotential (see the treatment in $[37,42]$, recently reviewed in $[70,74]$ ), and thus they respectively are an " $s$ polarized", "t-polarized" and " $u$-polarized" non-BPS $Z=0$ fake superpotential.

Eqs. (31), (34) and (35) can also be rewritten respectively as follows:

$$
\begin{aligned}
& \mathcal{W}_{\text {non-BPS,Z=0,s}}=\sqrt{g^{1 \overline{1}}\left(D_{s} Z\right) \overline{D_{\bar{s}}} \bar{Z}} ;
\end{aligned}
$$

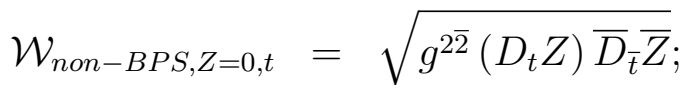

$$
\begin{aligned}
& \mathcal{W}_{\text {non }-B P S, Z=0, u}=\sqrt{g^{3 \overline{3}}\left(D_{u} Z\right) \bar{D}_{\bar{u}} \bar{Z}},
\end{aligned}
$$

where $D_{s} Z \equiv\left[\partial_{s}+\frac{1}{2}\left(\partial_{s} K\right)\right] Z$ is the covariant derivarive of $Z$ along the direction $s$ (and analogously for the moduli $t$ and $u$ ).

Due to the complete factorization of the manifold $\left(\frac{S U(1,1)}{U(1)}\right)^{3}$ (determining the diagonality of the metric $g^{i \bar{j}}$, given by the second Eq. of (4)), Eqs. (36), (38) and (37) are manifestly H-invariant. This result is consistent with the $H$-invariance imposed by the relation (given by Eq. (2.21) of [37], as well as by Eq. (13) of [42], and reported here for completeness' sake)

$$
\frac{d U(\tau, \Gamma)}{d \tau}=e^{U(\tau)} W(z(\tau), \bar{z}(\tau), \Gamma)
$$

between $\mathcal{W}$ and the warp factor $U(\tau)$ appearing in the Ansatz for the static, spherically symmetric, asymptotically flat, extremal dyonic $\mathrm{BH}$ metric:

$$
d s^{2}=-e^{2 U(\tau)} d t^{2}+e^{-2 U(\tau)} d \vec{x}^{2} .
$$

Actually, in the treatment given in the present paper, Eq. (39) has been crucial in order to guess (and thus check) the analytical form of the fake superpotential $\mathcal{W}(z(\tau), \bar{z}(\tau), \Gamma)$, by knowing the analyitical solution for the warp factor $U(\tau, \Gamma)$ relevant to the considered charge configuration.

Under the integrability conditions (13), the flow (29) is the most general solution (within the " $s$ polarization" defined by Eq. (26)) of the "non-BPS $Z=0$ analogue" of the $\frac{1}{2}$-BPS stabilization Eqs. (14), namely of:

$$
\mathcal{H}^{T}(\tau)=2 e^{K(z(\tau), \bar{z}(\tau))} \operatorname{Im}\left[g^{i \bar{j}}(z(\tau), \bar{z}(\tau))\left(\begin{array}{c}
\left(D_{i} X^{\Lambda}\right)(z(\tau), \bar{z}(\tau)) \\
\left(D_{i} F_{\Lambda}\right)(z(\tau), \bar{z}(\tau))
\end{array}\right)(\bar{\partial} \bar{j} \bar{W})(\bar{z}(\tau), \mathcal{H}(\tau))\right],
$$


which we can refer to as the non-BPS $Z=0$ stabilization Equations. It is easy realized that they can be obtained from the non-BPS $Z=0$ Attractor Eqs. (see the treatment in [23])

$$
\Gamma^{T}=2 e^{K(z, \bar{z})} \operatorname{Im}\left[g^{i \bar{j}}(z, \bar{z})\left(\begin{array}{c}
\left(D_{i} X^{\Lambda}\right)(z, \bar{z}) \\
\left(D_{i} F_{\Lambda}\right)(z, \bar{z})
\end{array}\right)\left(\bar{\partial}_{\bar{j}} \bar{W}\right)(\bar{z}, \Gamma)\right]
$$

by simply replacing $\Gamma$ with $\mathcal{H}(\tau)$. Consistently, Eq. (42) is the near-horizon $(\tau \rightarrow-\infty)$ limit of Eq. (41).

A remarkable consequence of the first order formalism for the non-BPS $Z=0$ attractor flow is that Eqs. (41) and (42) can actually be recast in the following $\left(\frac{1}{2}-\right)$ BPS-like forms in terms of the non-BPS $Z=0$ fake superpotential( $s$, respectively:

$$
\begin{aligned}
\mathcal{H}^{T}(\tau) & =2 e^{K(z(\tau), \bar{z}(\tau))} \operatorname{Im}\left[\mathfrak{W}_{\text {non-BPS,Z=0}}(z(\tau), \bar{z}(\tau), \mathcal{H}(\tau))\left(\begin{array}{c}
\bar{X}_{f}^{\Lambda}(z(\tau), \bar{z}(\tau)) \\
\bar{F}_{\Lambda, f}(z(\tau), \bar{z}(\tau))
\end{array}\right)\right] ; \\
\Gamma^{T} & =2 e^{K(z, \bar{z})} \operatorname{Im}\left[\mathfrak{W}_{\text {non-BPS,Z=0 }}(z, \bar{z}, \Gamma)\left(\begin{array}{c}
\bar{X}_{f}^{\Lambda}(z, \bar{z}) \\
\bar{F}_{\Lambda, f}(z, \bar{z})
\end{array}\right)\right],
\end{aligned}
$$

where

$$
\begin{aligned}
& \mathfrak{W}_{\text {non-BPS } Z=0}(z, \bar{z}, \Gamma)=Z(s, \bar{t}, \bar{u}, \Gamma) \text { in the "s-polarization" (Eq. 26); } \\
& \mathfrak{W}_{\text {non-BPS,Z=0}}(z, \bar{z}, \Gamma)=Z(\bar{s}, t, \bar{u}, \Gamma) \text { in the "t-polarization" (Eq. 32); } \\
& \mathfrak{W}_{\text {non-BPS,Z=0}}(z, \bar{z}, \Gamma)=Z(\bar{s}, \bar{t}, u, \Gamma) \text { in the "u-polarization" (Eq. 33), }
\end{aligned}
$$

such that in general

$$
\mathcal{W}_{\text {non }-B P S, Z=0}=\left|\mathfrak{W}_{\text {non }-B P S, Z=0}\right| \text {. }
$$

The subscript " $f$ " in Eqs. (43) and (44) indicates that a flipping of the dilatons has been performed ((Eqs. (26), (32) and (33), respectively for the choices (45), (46) and (47)). Notice that such a flipping destroys the holomorphicity of $X^{\Lambda}$ and $F_{\Lambda}$ in the moduli.

Eq. (29) is the most general solution of Eq. (43) with the choice (45), and equivalent expressions for the most general non-BPS $Z=0$ attractor flow can be obtained by solving Eq. (43) with the choice (46) or (47).

Such a $\left(\frac{1}{2}-\right)$ BPS-like reformulation of the non-BPS $Z=0$ stabilization Equations (41) and of their near-horizon $(\tau \rightarrow-\infty)$ limit given by the non-BPS $Z=0$ Attractor Eqs. (42) is possible due to the strict similarity between the most general $\frac{1}{2}$-BPS and non-BPS $Z=0$ attractor flows in the considered stu model, which actually are related through a flipping of two dilatons out of three. This in turn is related once again to the absence of flat directions along such flows, such that all moduli are explicitly determined as functions of dyonic BH charges (and as functions of $\tau$ ) all along the attractor flow.

Now, by exploiting the first order formalism [118] for $d=4$ extremal BHs [37, 42] (see also [70] and [74]), one can compute the relevant BH parameters of the non-BPS $Z=0$ attractor flow of the 
stu model starting from the expression of the non-BPS $Z=0$ fake superpotential $\mathcal{W}_{\text {non-BPS,Z=0 }}$ given by Eqs. (31), (34) or (35). The choice of "s-polarization", "t-polarization" or "u-polarization" is immaterial, due to the underlying triality symmetry of the moduli $s, t$ and $u$. Thus, without loss of generality, we choose to perform computations in the "s-polarization" (equivalent results in the other two "polarizations" can be obtained by cyclic permutations of the moduli).

Eqs. (31) and (19) yield the following expressions of the ADM mass of the non-BPS $Z=0$ attractor flow of the stu model:

$$
\begin{gathered}
M_{A D M, n o n-B P S, Z=0}\left(z_{\infty}, \bar{z}_{\infty}, \Gamma\right) \equiv \lim _{\tau \rightarrow 0^{-}} \mathcal{W}_{\text {non-BPS,Z=0,s }}(z(\tau), \bar{z}(\tau), \Gamma)= \\
=\lim _{\tau \rightarrow 0^{-}}|Z(s(\tau), \bar{t}(\tau), \bar{u}(\tau))|= \\
=\frac{\left|q_{0}+q_{1} s_{\infty}+q_{2} \bar{t}_{\infty}+q_{3} \bar{u}_{\infty}+p^{0} s_{\infty} \bar{t}_{\infty} \bar{u}_{\infty}-p^{1} \bar{t}_{\infty} \bar{u}_{\infty}-p^{2} s_{\infty} \bar{u}_{\infty}-p^{3} s_{\infty} \bar{t}_{\infty}\right|}{\sqrt{-i\left(s_{\infty}-\bar{s}_{\infty}\right)\left(t_{\infty}-\bar{t}_{\infty}\right)\left(u_{\infty}-\bar{u}_{\infty}\right)}}
\end{gathered}
$$

Eq. (49) yields that the marginal bound [111] is not saturated by non-BPS $Z=0$ states, because $M_{A D M, n o n-B P S, Z=0}$ is not equal to the sum of the $A D M$ masses of four D6-branes with appropriate fluxes (for further detail, see the discussion in [59]). This is actually expected, due to the strict similarity, discussed above, between $\frac{1}{2}$-BPS and non -BPS $Z=0$ attractor flows in the considered stu model; such a similarity can be explained by noticing that both such flows can be uplifted to the same $\frac{1}{8}$-BPS non-degenerate attractor flow of $\mathcal{N}=8, d=4$ supergravity (see e.g. the discussion in [50]).

Concerning the (covariant) scalar charges of the non-BPS $Z=0$ attractor flow of the stu model, they can be straightforwardly computed (in the "s-polarization", without loss of generality) by using Eqs. (31) and (20):

$$
\begin{aligned}
& \Sigma_{s, \text { non }-B P S, Z=0}\left(z_{\infty}, \bar{z}_{\infty}, \Gamma\right) \equiv \lim _{\tau \rightarrow 0^{-}}\left(\partial_{s} \mathcal{W}_{\text {non-BPS,Z=0,s}}\right)(z(\tau), \bar{z}(\tau), \Gamma)= \\
& =\lim _{\tau \rightarrow 0^{-}} \partial_{s}|Z(s(\tau), \bar{t}(\tau), \bar{u}(\tau))|= \\
& =\lim _{\tau \rightarrow 0^{-}} \frac{e^{K / 2}}{2}\left[\left(\partial_{s} K\right)|W(s, \bar{t}, \bar{u})|+\left(\partial_{s} W(s, \bar{t}, \bar{u})\right) \sqrt{\frac{\bar{W}(\bar{s}, t, u)}{W(s, \bar{t}, \bar{u})}}\right]= \\
& =\frac{1}{2 \sqrt{-i\left(s_{\infty}-\bar{s}_{\infty}\right)\left(t_{\infty}-\bar{t}_{\infty}\right)\left(u_{\infty}-\bar{u}_{\infty}\right)}} \cdot \\
& \cdot\left[\frac{\left|q_{0}+q_{1} s_{\infty}+q_{2} \bar{t}_{\infty}+q_{3} \bar{u}_{\infty}+p^{0} s_{\infty} \bar{t}_{\infty} \bar{u}_{\infty}-p^{1} \bar{t}_{\infty} \bar{u}_{\infty}-p^{2} s_{\infty} \bar{u}_{\infty}-p^{3} s_{\infty} \bar{t}_{\infty}\right|}{\left(s_{\infty}-\bar{s}_{\infty}\right)}+\right. \\
& +\left(q_{1}+p^{0} \bar{t}_{\infty} \bar{u}_{\infty}-p^{2} \bar{u}_{\infty}-p^{3} \bar{t}_{\infty}\right) \cdot \\
& \left.\cdot \sqrt{\frac{q_{0}+q_{1} \bar{s}_{\infty}+q_{2} t_{\infty}+q_{3} u_{\infty}+p^{0} \bar{s}_{\infty} t_{\infty} u_{\infty}-p^{1} t_{\infty} u_{\infty}-p^{2} \bar{s}_{\infty} u_{\infty}-p^{3} \bar{s}_{\infty} t_{\infty}}{q_{0}+q_{1} s_{\infty}+q_{2} \bar{t}_{\infty}+q_{3} \bar{u}_{\infty}+p^{0} s_{\infty} \bar{t}_{\infty} \bar{u}_{\infty}-p^{1} \bar{t}_{\infty} \bar{u}_{\infty}-p^{2} s_{\infty} \bar{u}_{\infty}-p^{3} s_{\infty} \bar{t}_{\infty}}}\right]= \\
& =\left.\Sigma_{s, \frac{1}{2}-B P S}\right|_{t_{\infty} \rightarrow \bar{t}_{\infty}, u_{\infty} \rightarrow \bar{u}_{\infty}} .
\end{aligned}
$$




$$
\begin{gathered}
\Sigma_{t, \text { non }-B P S, Z=0}\left(z_{\infty}, \bar{z}_{\infty}, \Gamma\right) \equiv \lim _{\tau \rightarrow 0^{-}}\left(\partial_{t} \mathcal{W}_{\text {non }-B P S, Z=0, s}\right)(z(\tau), \bar{z}(\tau), \Gamma)= \\
=\lim _{\tau \rightarrow 0^{-}} \partial_{t}|Z(s(\tau), \bar{t}(\tau), \bar{u}(\tau))|= \\
=\lim _{\tau \rightarrow 0^{-}} \frac{e^{K / 2}}{2}\left[\left(\partial_{t} K\right)|W(s, \bar{t}, \bar{u})|+\left(\partial_{t} \bar{W}(\bar{s}, t, u)\right) \sqrt{\left.\frac{W(s, \bar{t}, \bar{u})}{\bar{W}(\bar{s}, t, u)}\right]}=\right. \\
=\frac{1}{2 \sqrt{-i\left(s_{\infty}-\bar{s}_{\infty}\right)\left(t_{\infty}-\bar{t}_{\infty}\right)\left(u_{\infty}-\bar{u}_{\infty}\right)}} \cdot \\
\cdot\left[\frac{\left|q_{0}+q_{1} s_{\infty}+q_{2} \bar{t}_{\infty}+q_{3} \bar{u}_{\infty}+p^{0} s_{\infty} \bar{t}_{\infty} \bar{u}_{\infty}-p^{1} \bar{t}_{\infty} \bar{u}_{\infty}-p^{2} s_{\infty} \bar{u}_{\infty}-p^{3} s_{\infty} \bar{t}_{\infty}\right|}{\left(t_{\infty}-\bar{t}_{\infty}\right)}+\right. \\
\left.+\sqrt{\frac{q_{0}+q_{1} s_{\infty}+q_{2} \bar{t}_{\infty}+q_{3} \bar{u}_{\infty}+p^{0} s_{\infty} \bar{t}_{\infty} \bar{u}_{\infty}-p^{1} \bar{t}_{\infty} \bar{u}_{\infty}-p^{2} s_{\infty} \bar{u}_{\infty}-p^{3} s_{\infty} \bar{t}_{\infty}}{q_{0}+q_{1} \bar{s}_{\infty}+q_{2} t_{\infty}+q_{3} u_{\infty}+p^{0} \bar{s}_{\infty} t_{\infty} u_{\infty}-p^{1} t_{\infty} u_{\infty}-p^{2} \bar{s}_{\infty} u_{\infty}-p^{3} \bar{s}_{\infty} t_{\infty}}}\right] .
\end{gathered}
$$

$$
\begin{gathered}
\sum_{u, \text { non }-B P S, Z=0}\left(z_{\infty}, \bar{z}_{\infty}, \Gamma\right) \equiv \lim _{\tau \rightarrow 0^{-}}\left(\partial_{u} \mathcal{W}_{\text {non }-B P S, Z=0, s}\right)(z(\tau), \bar{z}(\tau), \Gamma)= \\
=\lim _{\tau \rightarrow 0^{-}} \partial_{u}|Z(s(\tau), \bar{t}(\tau), \bar{u}(\tau))|= \\
=\lim _{\tau \rightarrow 0^{-}} \frac{e^{K / 2}}{2}\left[\left(\partial_{u} K\right)|W(s, \bar{t}, \bar{u})|+\left(\partial_{u} \bar{W}(\bar{s}, t, u)\right) \sqrt{\left.\frac{W(s, \bar{t}, \bar{u})}{\bar{W}(\bar{s}, t, u)}\right]}=\right. \\
=\frac{1}{2 \sqrt{-i\left(s_{\infty}-\bar{s}_{\infty}\right)\left(t_{\infty}-\bar{t}_{\infty}\right)\left(u_{\infty}-\bar{u}_{\infty}\right)}} \cdot \\
\cdot\left[\frac{\left|q_{0}+q_{1} s_{\infty}+q_{2} \bar{t}_{\infty}+q_{3} \bar{u}_{\infty}+p^{0} s_{\infty} \bar{t}_{\infty} \bar{u}_{\infty}-p^{1} \bar{t}_{\infty} \bar{u}_{\infty}-p^{2} s_{\infty} \bar{u}_{\infty}-p^{3} s_{\infty} \bar{t}_{\infty}\right|}{\left(u_{\infty}-\bar{u}_{\infty}\right)}+\right. \\
\left.+\sqrt{\frac{q_{0}+q_{1} s_{\infty}+q_{2} \bar{t}_{\infty}+q_{3} \bar{u}_{\infty}+p^{0} s_{\infty} \bar{t}_{\infty} \bar{u}_{\infty}-p^{1} \bar{t}_{\infty} \bar{u}_{\infty}-p^{2} s_{\infty} \bar{u}_{\infty}-p^{3} s_{\infty} \bar{t}_{\infty}}{q_{0}+q_{1} \bar{s}_{\infty}+q_{2} t_{\infty}+q_{3} u_{\infty}+p^{0} \bar{s}_{\infty} t_{\infty} u_{\infty}-p^{1} t_{\infty} u_{\infty}-p^{2} \bar{s}_{\infty} u_{\infty}-p^{3} \bar{s}_{\infty} t_{\infty}}}\right] .
\end{gathered}
$$

Also, it is here worth computing the difference between the squared non-BPS $Z=0$ fake superpotential and the squared absolute value of the $\mathcal{N}=2, d=4$ central charge along the considered non-BPS $Z=0$ attractor flow. This amounts to computing the difference generalizing the BPS bound [112] to the 
whole attractor flow (without loss of generality, we perform calculations in the "s-polarization"):

$$
\begin{aligned}
& \Theta(\mathcal{X}, \mathcal{Y}, \Gamma) \equiv \mathcal{W}_{s, \text { non }-B P S, Z=0}^{2}-|Z|^{2}= \\
= & -\frac{1}{8\left(p^{1} p^{3}-p^{0} q_{2}\right) \mathcal{Y}^{2}}\left[\left(p^{\Lambda} q_{\Lambda}-2 p^{2} q_{2}-2\left(p^{1} p^{3}-p^{0} q_{2}\right) \mathcal{X}^{2}\right)^{2}+4\left(p^{1} p^{3}-p^{0} q_{2}\right)^{2}\left(\mathcal{Y}^{2}\right)^{2}+\mathcal{I}_{4}\right]- \\
& -\frac{1}{8\left(p^{1} p^{2}-p^{0} q_{3}\right) \mathcal{Y}^{2}}\left[\left(p^{\Lambda} q_{\Lambda}-2 p^{3} q_{3}-2\left(p^{1} p^{2}-p^{0} q_{3}\right) \mathcal{X}^{3}\right)^{2}+4\left(p^{1} p^{2}-p^{0} q_{3}\right)^{2}\left(\mathcal{Y}^{2}\right)^{2}+\mathcal{I}_{4}\right]>0 .
\end{aligned}
$$

Such an expression for the scalar-dependent, strictly positive $\Theta$ was obtained by using the following relations:

$$
\begin{aligned}
& \left(p^{\Lambda} q_{\Lambda}-2 p^{2} q_{2}\right)^{2}+\mathcal{I}_{4}=4\left(p^{1} p^{3}-p^{0} q_{2}\right)\left(q_{1} q_{3}+p^{2} q_{0}\right) \\
& \left(p^{\Lambda} q_{\Lambda}-2 p^{3} q_{3}\right)^{2}+\mathcal{I}_{4}=4\left(p^{1} p^{2}-p^{0} q_{3}\right)\left(q_{1} q_{2}+p^{3} q_{0}\right) .
\end{aligned}
$$

Thus, the BPS bound [112] holds not only at the BH event horizon $\left(r=r_{H}\right)$, but actually (in a scalardependent way) all along the non-BPS $Z=0$ attractor flow (i.e. $\forall r \geqslant r_{H}$ ).

\section{The most General Non-BPS $Z \neq 0$ Attractor Flow}

All the features holding for $\frac{1}{2}$-BPS and non-BPS $Z=0$ attractor flows (respectively treated in Sects. 3. and 4.) do not directly hold for the non-BPS $Z \neq 0$ attractor flow, which actually turns out to be rather different from (and structurally much more intricate than) such two attractor flows.

As mentioned in the Introduction, the non-BPS $Z \neq 0$ attractor flow of the stu model has been already considered in literature in particular cases, namely for the D0 - D4 (magnetic) [51, 59], D0 - D6 [59], D2 - D6 (electric) [28, 62] D0 - D2 - D4 (magnetic with D2) [62], D0 - D2 - D4 - D6 (without $B$-fields) [28] supporting BH charge configurations.

In the present Section we determine the explicit expression of the non-BPS $Z \neq 0$ attractor flow for the most general supporting $\mathrm{BH}$ charge configuration, with all electric and magnetic charges switched on, namely for the non-BPS $Z \neq 0$-supporting branch of the $D 0-D 2-D 4-D 6$ configuration. Thence, as already done for $\frac{1}{2}$-BPS and non-BPS $Z=0$ attractor flows, by exploiting the first order (fake supergravity) formalism [37, 42, 118], we compute the ADM masses as well as the covariant scalar charges, and study the issue of marginal stability [111], completing and refining the treatment given in $[51,59,62]$.

\subsection{U-Duality Transformations along the Orbit $\mathcal{O}_{\text {non-BPS,Z } \neq 0}$}

In order to derive the explicit expression of the non-BPS $Z \neq 0$ attractor flow when all $\mathrm{BH}$ charges are non-vanishing, we exploit a method already used in [28], [59] and [62], based on performing suitable symplectic transformations along the relevant (i.e. supporting) charge orbit of the $U$-duality group. In Eqs. (16) and (28) we recalled the form of the $\frac{1}{2}$-BPS- and non-BPS $Z=0$ - supporting BH charge orbits of the tri-fundamental representation $(2,2,2)$ of the $U$-duality group $G$ (given by Eq.(1)) of the stu model, also commenting on their separation [26]. The corresponding non-BPS $Z \neq 0$-supporting $\mathrm{BH}$ charge orbit reads [26]

$$
\mathcal{O}_{n o n-B P S, Z \neq 0}=\frac{(S U(1,1))^{3}}{(S O(1,1))^{2}},
$$


defined by the constraint

$$
\mathcal{I}_{4}(\Gamma)<0 .
$$

As done in [59] and [62], in order to perform a symplectic transformation along the charge orbit

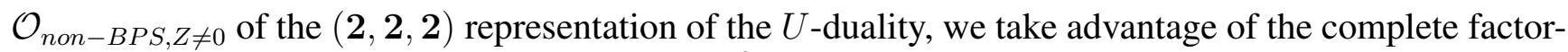
ization of the special Kähler manifold $\left(\frac{S U(1,1)}{U(1)}\right)^{3}$ (ultimately determining the triality symmetry), which allows one to deal with the product of three distinct $2 \times 2$ matrices of $S L(2, \mathbb{R})$, rather than with a unique $8 \times 8$ matrix of the $U$-duality group embedded in the relevant symplectic group $S p(8, \mathbb{R})$.

The first step is to perform an $S p(8, \mathbb{R})$-transformation from the basis $\left(p^{\Lambda}, q_{\Lambda}\right)$ to a basis $\mathcal{A}_{a b c}$ ( $a, b, c=0,1$ throughout) of $\mathrm{BH}$ charges expicitly transforming under the $(\mathbf{2}, \mathbf{2}, \mathbf{2})$ of the $U$-duality. Such a transformation is given by Eq. (5.1) of [59] (equivalent to Eq. (3.5) of the second Ref. of [3]; see also Section 5 of [50]). The explicit action of a generic symplectic transformation of the $U$-duality on the $\mathrm{BH}$ charges $\mathcal{A}_{a b c}$ is given, up to some change of notation, by Eqs. (5.2) and (5.3) of [59], which we report below for simplicity's sake:

$$
\begin{aligned}
\mathcal{A}_{a^{\prime} b^{\prime} c^{\prime}}^{\prime} & =\left(M_{1}\right)_{a^{\prime}}^{a}\left(M_{2}\right)_{b^{\prime}}^{b}\left(M_{3}\right)_{c^{\prime}}^{c} a_{a b c} ; \\
M_{i} & \equiv\left(\begin{array}{cc}
\mathfrak{a}_{i} & \mathfrak{b}_{i} \\
\mathfrak{c}_{i} & \mathfrak{d}_{i}
\end{array}\right) \in S L(2, \mathbb{R}), \operatorname{det}\left(M_{i}\right)=1, \forall i=1,2,3,
\end{aligned}
$$

where each matrix pertains to the degrees of freedom of only one modulus (e.g. $M_{1}$ to $s, M_{2}$ to $t, M_{3}$ to $u$ ). The transformation (57)-(58) of $(S L(2, \mathbb{R}))^{3} \subset S p(8, \mathbb{R})$ induces also a linear fractional (Möbius) transformation on the moduli $z^{i}$ as follows (no summation on repeated indices; once again, we report Eq. (5.3) of [59] for simplicity's sake; also recall Eq. (2)):

$$
z^{i}=\frac{\mathfrak{a}_{i} z^{i}+\mathfrak{b}_{i}}{\mathfrak{c}_{i} z^{i}+\mathfrak{d}_{i}}
$$

As done in [59] and [62], we use the configuration $D 0-D 6$ as "pivot" in order to perform the transformation (57)-(59). Indeed, such a BH charge configuration supports only non-BPS $Z \neq 0$ attractors, as it can be easily realized by computing the corresponding quartic $U$-invariant, given by Eq. (8): $\mathcal{I}_{4}\left(\Gamma_{D 0-D 6}\right)<0$ (see also the treatment of [47]). Thus, we want to transform from the configuration $D 0-D 6$ (corresponding to charges $\left(q_{0}, p^{0}\right)$, which we denote here $(q, p)$ ) to the most general configuration $D 0-D 2-D 4-D 6$, corresponding to all $\mathrm{BH}$ charges switched on: $\left(q_{0}, q_{i}, p^{i}, p^{0}\right)$. By exploiting the transformation (57)-(59), the parameters $\mathfrak{a}_{i}, \mathfrak{b}_{i}, \mathfrak{c}_{i}, \mathfrak{d}_{i}$ of the $M_{i}$ s dualizing from $D 0-D 6$ to $D 0-D 2-D 4-D 6$ must satisfy the following set of constraints:

$$
\begin{aligned}
-q_{0} & =-\mathfrak{a}_{1} \mathfrak{a}_{2} \mathfrak{a}_{3} q+\mathfrak{b}_{1} \mathfrak{b}_{2} \mathfrak{b}_{3} p \\
q_{i} & =-\frac{1}{2} s_{i j k} \mathfrak{c}_{i} \mathfrak{a}_{j} \mathfrak{a}_{k} q+\frac{1}{2} s_{i j k} \mathfrak{d}_{i} \mathfrak{b}_{j} \mathfrak{b}_{k} p \\
p^{i} & =-\frac{1}{2} s_{i j k} \mathfrak{a}_{i} \mathfrak{c}_{j} \mathfrak{c}_{k} q+\frac{1}{2} s_{i j k} \mathfrak{b}_{i} \mathfrak{d}_{j} \mathfrak{d}_{k} p \\
p^{0} & =-\mathfrak{c}_{1} \mathfrak{c}_{2} \mathfrak{c}_{3} q+\mathfrak{d}_{1} \mathfrak{d}_{2} \mathfrak{d}_{3} p
\end{aligned}
$$


where $s_{i j k} \equiv\left|\epsilon_{i j k}\right|$. Notice that the system (60) admits solutions iff the condition (56) is met; this implies the transformation (57)-(59) to belong to the $U$-duality orbit $\mathcal{O}_{n o n-B P S, Z \neq 0}$ given by Eq. (55). The sign of the BH charges $q$ and $p$ is actually irrelevant for the condition (56) to be satisfied; thus, without loss of any generality, one can choose $e . g . q>0, p>0$. Within such a choice, the explicit form of the matrices $M_{i}$ s under consideration (and of their inverse) reads as follows:

$$
\begin{aligned}
M_{i} & =-\frac{\operatorname{sgn}(\xi)}{\sqrt{\left(\varsigma_{i}+\varrho_{i}\right) \xi}}\left(\begin{array}{cc}
\varsigma_{i} \xi & -\varrho_{i} \\
\xi & 1
\end{array}\right) \Leftrightarrow M_{i}^{-1}=-\frac{\operatorname{sgn}(\xi)}{\sqrt{\left(\varsigma_{i}+\varrho_{i}\right) \xi}}\left(\begin{array}{cc}
1 & \varrho_{i} \\
-\xi & \varsigma_{i} \xi
\end{array}\right) ; \\
\xi & \equiv\left(\frac{p}{q}\right)^{1 / 3}\left[\frac{2 p^{1} p^{2} p^{3}+p^{0}\left(\sqrt{-\mathcal{I}_{4}}-p^{\Lambda} q_{\Lambda}\right)}{2 p^{1} p^{2} p^{3}-p^{0}\left(\sqrt{-\mathcal{I}_{4}}-p^{\Lambda} q_{\Lambda}\right)}\right]^{1 / 3} \in \mathbb{R} ; \\
\varsigma_{i} & \equiv \frac{\sqrt{-\mathcal{I}_{4}}+p^{\Lambda} q_{\Lambda}-2 p^{i} q_{i}}{s_{i j k} p^{j} p^{k}-2 p^{0} q_{i}} \in \mathbb{R}(\text { no sum on } i) ; \\
\varrho_{i} & \equiv \frac{\sqrt{-\mathcal{I}_{4}}-p^{\Lambda} q_{\Lambda}+2 p^{i} q_{i}}{s_{i j k} p^{j} p^{k}-2 p^{0} q_{i}} \in \mathbb{R}(\text { no sum on } i) .
\end{aligned}
$$

The definitions (63) and (64), together with Eq. (8), imply that (no sum on $i$ )

$$
\varsigma_{i} \varrho_{i}=-\frac{s_{i j k} q_{j} q_{k}+2 q_{0} p^{i}}{s_{i j k} p^{j} p^{k}-2 p^{0} q_{i}} .
$$

As expected since the transformation (57)-(59) belongs to the orbit $\mathcal{O}_{n o n-B P S, Z \neq 0}$ of the $U$-duality, it leaves $\mathcal{I}_{4}$ unchanged:

$\mathcal{I}_{4}\left(\Gamma_{D 0-D 2-D 4-D 6}\right)=-\left(p^{\Lambda} q_{\Lambda}\right)^{2}+4 \sum_{i<j} p^{i} q_{i} p^{j} q_{j}-4 p^{0} q_{1} q_{2} q_{3}+4 q_{0} p^{1} p^{2} p^{3}=-(p q)^{2}=\mathcal{I}_{4}\left(\Gamma_{D 0-D 6}\right)$.

It should be stressed that the transformation (57)-(59) (along with Eqs. (61)-(64)) is not the most general transformation of $\mathcal{O}_{n o n-B P S, Z \neq 0}$ mapping the $D 0-D 6$ into the $D 0-D 2-D 4-D 6$ configuration (and vice versa). Indeed, it may be further generalized by replacing $\xi$ with a triplet $\xi_{i}$, constrained by $\xi_{1} \xi_{2} \xi_{3}=\xi^{3}$. Such a two-parameter generalization of the above transformation indicates, as mentioned above, the presence of a real, 2-dim. moduli space (namely $\left.(S O(1,1))^{2}[41,43]\right)$ all along the non$B P S Z \neq 0$ attractor flow; this will become evident when looking at the explicit form of such a flow, presented further below.

\subsection{D0 - D6: the Most General Flow and Fake Superpotential}

The most general non-BPS $Z \neq 0$ attractor flow in the $D 0-D 6$ configuration reads as follows [59]:

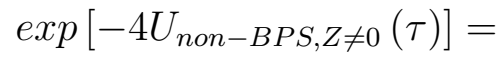

$$
\begin{aligned}
& =\left[a-\left(-\mathcal{I}_{4}\right)^{1 / 4} \tau\right]\left[k^{1}-\left(-\mathcal{I}_{4}\right)^{1 / 4} \tau\right]\left[k^{2}-\left(-\mathcal{I}_{4}\right)^{1 / 4} \tau\right]\left[k^{3}-\left(-\mathcal{I}_{4}\right)^{1 / 4} \tau\right]-b^{2} ;
\end{aligned}
$$




$$
\begin{aligned}
& x_{n o n-B P S, Z \neq 0}^{i}(\tau)=\xi_{0}^{-1} e^{\alpha_{i}} . \\
& \cdot \frac{\left[k^{j}-\left(-\mathcal{I}_{4}\right)^{1 / 4} \tau\right]\left[k^{l}-\left(-\mathcal{I}_{4}\right)^{1 / 4} \tau\right]-\left[a-\left(-\mathcal{I}_{4}\right)^{1 / 4} \tau\right]\left[k^{i}-\left(-\mathcal{I}_{4}\right)^{1 / 4} \tau\right]}{\left[k^{j}-\left(-\mathcal{I}_{4}\right)^{1 / 4} \tau\right]\left[k^{l}-\left(-\mathcal{I}_{4}\right)^{1 / 4} \tau\right]+\left[a-\left(-\mathcal{I}_{4}\right)^{1 / 4} \tau\right]\left[k^{i}-\left(-\mathcal{I}_{4}\right)^{1 / 4} \tau\right]-2 b} \\
& y_{n o n-B P S, Z \neq 0}^{i}(\tau)=2 \xi_{0}^{-1} e^{\alpha_{i}} .
\end{aligned}
$$

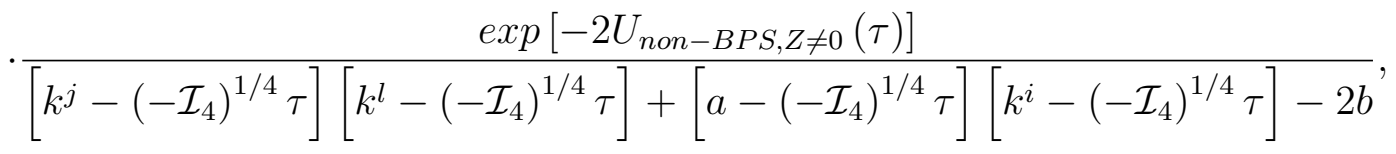

where

$$
\xi_{0} \equiv(p / q)^{1 / 3}
$$

$a \in \mathbb{R}_{0}, b, k^{i} \in \mathbb{R}\left(k^{i}\right.$ s cannot all vanish), and the triplet of real constants $\alpha_{i}$ satisfies the constraint

$$
\sum_{i} \alpha_{i}=0
$$

It is worth pointing out that the $D 0-D 6$ configuration supports axion-free non-BPS $Z \neq 0$ attractor flow(s); when considering the near-horizon limit, and thus the critical, charge-dependent values of the moduli, this is consistent with the analysis performed in [15, 41, 47]. An axion-free attractor flow solution of Eqs. (67)-(69) can be obtained e.g. by putting

$$
k^{i}=a \forall i=1,2,3,
$$

and it reads as follows:

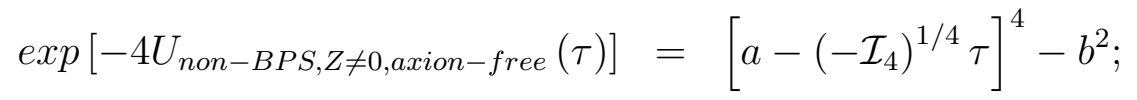

$$
\begin{aligned}
& x_{\text {non-BPS,Z} \neq \neq 0, \text { axion-free }}^{i}(\tau)=0 \text {; } \\
& y_{\text {non-BPS,Z } \neq 0, \text { axion-free }}^{i}(\tau)=\xi_{0}^{-1} e^{\alpha_{i}} \sqrt{\frac{\left[a-\left(-\mathcal{I}_{4}\right)^{1 / 4} \tau\right]^{2}+b}{\left[a-\left(-\mathcal{I}_{4}\right)^{1 / 4} \tau\right]^{2}-b}} .
\end{aligned}
$$

The non-BPS $Z \neq 0$ fake superpotential of the first order formalism can be computed to have the following form in the $D 0-D 6$ configuration:

$$
\begin{aligned}
& \mathcal{W}_{\text {non-BPS }, Z \neq 0}(z, \bar{z}, q, p)=\frac{1}{4} e^{K / 2}\left[\prod_{i}\left|q^{1 / 3}+p^{1 / 3} e^{-\alpha_{i}} z^{i}\right|\right] \\
& {\left[1+\sum_{i<j} \frac{\left(q^{2 / 3}-p^{2 / 3} e^{-2 \alpha_{i}}\left|z^{i}\right|^{2}\right)\left(q^{2 / 3}-p^{2 / 3} e^{-2 \alpha_{j}}\left|z^{j}\right|^{2}\right)-e^{-\alpha_{i}-\alpha_{j}} q^{2 / 3} p^{2 / 3}\left(z^{i}-\bar{z}^{\bar{i}}\right)\left(z^{j}-\bar{z}^{\bar{j}}\right)}{\left|q^{1 / 3}+p^{1 / 3} e^{-\alpha_{i}} z^{i}\right|^{2}\left|q^{1 / 3}+p^{1 / 3} e^{-\alpha_{j}} z^{j}\right|^{2}}\right] .}
\end{aligned}
$$


The axion-free version of such a fake superpotential (e.g. pertaining to the solution (73)-(73)) reads as follows:

$$
\begin{aligned}
& \mathcal{W}_{\text {non-BPS,Z } \neq 0, \text { axion-free }}(y, q, p)=\frac{1}{2^{3} \sqrt{2}} \frac{1}{\sqrt{y^{1} y^{2} y^{3}}}\left[\prod_{l}\left|q^{1 / 3}-i p^{1 / 3} e^{-\alpha_{l}} y^{l}\right|\right] . \\
& {\left[1+\sum_{i<j} \frac{\left[q^{2 / 3}-p^{2 / 3} e^{-2 \alpha_{i}}\left(y^{i}\right)^{2}\right]\left[q^{2 / 3}-p^{2 / 3} e^{-2 \alpha_{j}}\left(y^{j}\right)^{2}\right]+4 e^{-\alpha_{i}-\alpha_{j}} q^{2 / 3} p^{2 / 3} y^{i} y^{j}}{\left|q^{1 / 3}-i p^{1 / 3} e^{-\alpha_{i}} y^{i}\right|^{2}\left|q^{1 / 3}-i p^{1 / 3} e^{-\alpha_{j}} y^{j}\right|^{2}}\right] .}
\end{aligned}
$$

Now, by exploiting the first order formalism [118] for $d=4$ extremal BHs [37, 42] (see also [70] and [74]), one can compute the relevant BH parameters of the non-BPS $Z \neq 0$ attractor flow of $d=4$ stu model in the $D 0-D 6$ configuration, starting from the expression of the non-BPS $Z \neq 0$ fake superpotential $\mathcal{W}_{\text {non-BPS,Z } \neq 0}$ given by Eq. (74).

Eqs. (74) and (19) yield, after some algebra, the following expression for the ADM mass:

$$
\begin{gathered}
M_{A D M, n o n-B P S, Z \neq 0}\left(z_{\infty}, \bar{z}_{\infty}, \Gamma_{D 0-D 6}\right)=\frac{P}{2^{7 / 2}}\left[\prod_{i} \sqrt{\left[\left(\Lambda^{i}\right)^{-1}+B^{i}\right]^{2}+1}\right] . \\
\cdot\left\{1+\sum_{i<j} \frac{\left[\left(\Lambda^{i}\right)^{-2}-\left(B^{i}\right)^{2}-1\right]\left[\left(\Lambda^{j}\right)^{-2}-\left(B^{j}\right)^{2}-1\right]+4\left(\Lambda^{i}\right)^{-1}\left(\Lambda^{j}\right)^{-1}}{\left[\left[\left(\Lambda^{i}\right)^{-1}+B^{i}\right]^{2}+1\right]\left[\left[\left(\Lambda^{j}\right)^{-1}+B^{j}\right]^{2}+1\right]}\right\},
\end{gathered}
$$

where the quantities

$$
\Lambda^{i} \equiv \xi_{0} y_{\infty}^{i} ; \quad B^{i} \equiv \frac{x_{\infty}^{i}}{y_{\infty}^{i}}, \quad P \equiv p \sqrt{y_{\infty}^{1} y_{\infty}^{2} y_{\infty}^{3}}, \quad Q \equiv \frac{q}{\sqrt{y_{\infty}^{1} y_{\infty}^{2} y_{\infty}^{3}}}
$$

were introduced, and, for simplicity's sake, the $\alpha_{i}$ s were chosen all to vanish (i.e. $\alpha_{i}=0 \forall i=1,2,3$ ). $P$ and $Q$ are the dressed charges, i.e. a sort of asymptotical redefinition of the charges pertaining to D6 and $D 0$ branes, respectively. On the other hand, $\Lambda^{i}$ and $B^{i}$ are usually named (asymptotical brane) fluxes and $B$-fields, respectively.

Eq. (5.2.) (along with the definitions (77)) coincides with Eq. (5.56) of [59], provided that the following condition is met (see Eq. (5.44) of [59]):

$$
\Lambda^{1}\left[1+\left(B^{1}\right)^{2}\right]-\left(\Lambda^{1}\right)^{-1}=\Lambda^{2}\left[1+\left(B^{2}\right)^{2}\right]-\left(\Lambda^{2}\right)^{-1}=\Lambda^{3}\left[1+\left(B^{3}\right)^{2}\right]-\left(\Lambda^{3}\right)^{-1} .
$$

As observed in [59], Eq. (5.2.) (along with the definitions (77)) yields the marginal bound [111] to be saturated, because $M_{A D M, n o n-B P S, Z \neq 0}$ is equal to the sum of the ADM masses of four D6-branes with appropriate fluxes (for further detail, see the discussion in [59]).

Concerning the (covariant) scalar charges, they can be straightforwardly computed by recalling Eqs. (74) and (20), but their expressions are rather cumbersome. For simplicity's sake, here we limit ourselves to give the scalar charges for the " $t^{3}$-degeneration" of the $s t u$ model (in which all charges and moduli are equal, insensitive to $i$-index; see e.g. Eq. (116) below, as well as the treatment in Sect. 5 of [50]). By denoting $t \equiv x-i y$, the covariant scalar charges of axion and dilaton in the $D 0-D 6$ configuration 
respectively read

$$
\begin{aligned}
\Sigma_{x, \text { non }-B P S, Z \neq 0}\left(x_{\infty}, y_{\infty}, \Gamma_{D 0-D 6}\right) & =\sqrt{2} P x_{\infty} \frac{\left(\Lambda^{-1} B+B^{2}+1\right)}{\sqrt{\left(\Lambda^{-1}+B\right)^{2}+1}} ; \\
\Sigma_{y, \text { non }-B P S, Z \neq 0}\left(x_{\infty}, y_{\infty}, \Gamma_{D 0-D 6}\right) & =-\frac{P y_{\infty}}{\sqrt{2}} \frac{\left[\Lambda^{-4}+\Lambda^{-3} B+\Lambda^{-1} B\left(B^{2}-1\right)+B^{4}-1\right]}{\sqrt{\left(\Lambda^{-1}+B\right)^{2}+1}} .
\end{aligned}
$$

\subsection{D0 - D2 - D4 - D6: the Most General Flow and Fake Superpotential}

Now, by performing the $U$-duality transformation (57-(59) (along with Eqs. (61)-(65)) and using the most general non-BPS $Z \neq 0$ attractor flow in the $D 0-D 6$ configuration given by Eqs. (67)-(69), it is a matter of long but straightforward computations to determine the most general non-BPS $Z \neq 0$ attractor flow in the most general configuration, namely in the $D 0-D 2-D 4-D 6$ one, in which all $\mathrm{BH}$ charges are switched on. It reads as follows (the moduli are here denoted as $\mathcal{Z}^{i} \equiv \mathcal{X}^{i}-i \mathcal{Y}^{i} ; i \neq j \neq l$ and no sum on repeated $i$-indices throughout):

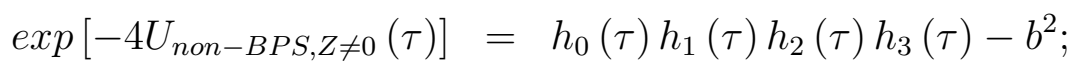

$$
\begin{aligned}
& \mathcal{X}_{n o n-B P S, Z \neq 0}^{i}(\tau)=\frac{\left\{\begin{array}{l}
\varsigma_{i} e^{2 \alpha_{i}} \nu^{2}\left[h_{j}(\tau) h_{l}(\tau)+h_{0}(\tau) h_{i}(\tau)+2 b\right]+ \\
+e^{\alpha_{i}} \nu\left(\varsigma_{i}-\varrho_{i}\right)\left[h_{j}(\tau) h_{l}(\tau)-h_{0}(\tau) h_{i}(\tau)\right]+ \\
-\varrho_{i}\left[h_{j}(\tau) h_{l}(\tau)+h_{0}(\tau) h_{i}(\tau)-2 b\right]
\end{array}\right\}}{\left\{\begin{array}{l}
e^{2 \alpha_{i}} \nu^{2}\left[h_{j}(\tau) h_{l}(\tau)+h_{0}(\tau) h_{i}(\tau)+2 b\right]+ \\
+2 e^{\alpha_{i}} \nu\left[h_{j}(\tau) h_{l}(\tau)-h_{0}(\tau) h_{i}(\tau)\right]+ \\
+h_{j}(\tau) h_{l}(\tau)+h_{0}(\tau) h_{i}(\tau)-2 b
\end{array}\right\}}
\end{aligned}
$$

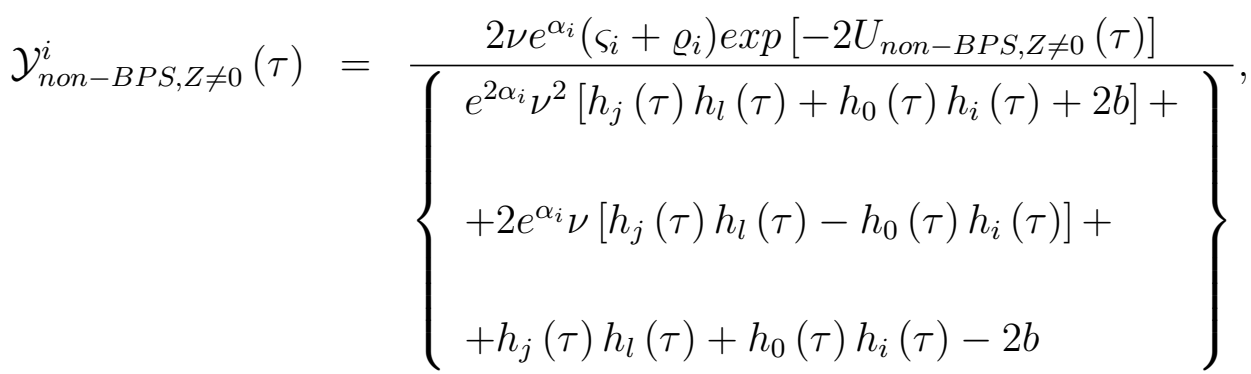


where $\varsigma_{i}$ and $\varrho_{i}$ have been defined in Eqs. (63) and (64), respectively. The constants $\alpha_{i}$ and $b$ have been introduced in Eqs. (67)-(69). Furthermore, the new quantities (see Eqs. (62) and (70), as well)

$$
\begin{aligned}
\nu & \equiv \frac{\xi}{\xi_{0}}=\left[\frac{2 p^{1} p^{2} p^{3}+p^{0}\left(\sqrt{-\mathcal{I}_{4}}-p^{\Lambda} q_{\Lambda}\right)}{2 p^{1} p^{2} p^{3}-p^{0}\left(\sqrt{-\mathcal{I}_{4}}-p^{\Lambda} q_{\Lambda}\right)}\right]^{1 / 3} \in \mathbb{R} ; \\
h_{\Lambda}(\tau) & \equiv b_{\Lambda}-\left(-\mathcal{I}_{4}\right)^{1 / 4} \tau,
\end{aligned}
$$

where $b_{\Sigma}$ are real constants, have been defined.

In the particular case in which $b=0$, the expression of $\exp \left[-4 U_{n o n-B P S, Z \neq 0}(\tau)\right]$ can be recast in the form

$$
\exp \left[-4 U_{\text {non }-B P S, Z \neq 0}(\tau)\right]=-\mathcal{I}_{4}(\mathcal{H}(\tau)),
$$

consistently with the results of [28] and [59], and on the same ground of (the first of) Eqs. (9) and (29), respectively holding for the $\frac{1}{2}$-BPS and non-BPS $Z=0$ attractor flows.

By performing the near-horizon (i.e. $\tau \rightarrow-\infty$ ) limit, Eqs. (82) and (83) respectively yield the following critical values of the moduli (the subscript " $H$ " stands for "horizon"):

$$
\begin{aligned}
& \mathcal{X}_{n o n-B P S, Z \neq 0, H}^{i} \equiv \lim _{\tau \rightarrow-\infty} \mathcal{X}_{n o n-B P S, Z \neq 0}^{i}(\tau)=\frac{\varsigma_{i} e^{2 \alpha_{i}} \nu^{2}-\varrho_{i}}{e^{2 \alpha_{i}} \nu^{2}+1} ; \\
& \mathcal{Y}_{n o n-B P S, Z \neq 0, H}^{i} \equiv \lim _{\tau \rightarrow-\infty} \mathcal{Y}_{n o n-B P S, Z \neq 0}^{i}(\tau)=\frac{1}{2} \frac{e^{\alpha_{i}}\left(\varsigma_{i}+\varrho_{i}\right) \nu}{e^{2 \alpha_{i}} \nu^{2}+1}=\frac{\sqrt{-\mathcal{I}_{4}} e^{\alpha_{i}} \nu}{\left(s_{i j k} p^{j} p^{k}-2 p^{0} q_{i}\right)\left(e^{2 \alpha_{i}} \nu^{2}+1\right)}
\end{aligned}
$$

It is worth pointing out that the $D 0-D 2-D 4-D 6$ configuration does not support axion-free non-BPS $Z \neq 0$ attractor flow(s); when considering the near-horizon limit, and thus the critical, charge-dependent values of the moduli, this is consistent with the analysis performed in [15, 41, 47].

The solution (81)-(83) (along with the definitions (84) and (85)) generalizes the result of [28]. As mentioned in the Introduction, in [28] it was shown that, within the (non-BPS $Z \neq 0$-supporting branches of the) $D 2-D 6$ (electric) and $D 0-D 2-D 4-D 6$ configurations, in absence of (some of the) $B$ fields the attractor flow solution can be obtained by replacing the $S p(8, \mathbb{R})$-covariant vector $\Gamma$ of charges (defined by Eq. (7)) with the $S p(8, \mathbb{R})$-covariant vector $\mathcal{H}(\tau)$ of harmonic functions (defined by Eqs. (10)-(13)) in the corresponding critical, horizon solution.

For the $\frac{1}{2}$-BPS and non-BPS $Z=0$ attractor flows, respectively treated in Sects. 3. and 4., such a procedure allows one to determine the most general attractor flow solution starting from the corresponding critical, horizon solution.

On the other hand, for the non-BPS $Z \neq 0$ attractor flow such a procedure fails in presence of nonvanishing $B$-fields. In other words, it can be shown that the completely general non-BPS $Z \neq 0$ attractor flow solution (81)-(83) is not a solution of the would-be non-BPS $Z \neq 0$ stabilization Eqs. (see e.g. the 
treatments of [23], [19] and [32])

$$
\begin{aligned}
\mathcal{H}^{T}(\tau)= & 2 e^{K(z(\tau), \bar{z}(\tau))} \operatorname{Im}\left[W(z(\tau))\left(\begin{array}{c}
\bar{X}^{\Lambda}(\bar{z}(\tau)) \\
\bar{F}_{\Lambda}(\bar{z}(\tau))
\end{array}\right)+\right. \\
& +\frac{i}{2} \frac{\bar{W}(\bar{z}(\tau))}{|W|^{2}(z(\tau), \bar{z}(\tau))} \bar{C}_{\overline{i j k}}(z(\tau), \bar{z}(\tau)) g^{i \bar{i}}(z(\tau), \bar{z}(\tau)) g^{j \bar{j}}(z(\tau), \bar{z}(\tau)) g^{k \bar{k}}(z(\tau), \bar{z}(\tau)) \cdot \\
& \left.\cdot\left(D_{j} W\right)(z(\tau), \bar{z}(\tau), \mathcal{H}(\tau))\left(D_{k} W\right)(z(\tau), \bar{z}(\tau), \mathcal{H}(\tau))\left(\begin{array}{c}
\left(D_{i} X^{\Lambda}\right)(z(\tau), \bar{z}(\tau)) \\
\left(D_{i} F_{\Lambda}\right)(z(\tau), \bar{z}(\tau))
\end{array}\right)\right],
\end{aligned}
$$

which can be obtained from the non-BPS $Z \neq 0$ Attractor Eqs. (see e.g. the treatments in [23] and [32])

$$
\begin{aligned}
\Gamma^{T}= & 2 e^{K(z, \bar{z})} \operatorname{Im}\left[W(z)\left(\begin{array}{c}
\bar{X}^{\Lambda}(\bar{z}) \\
\bar{F}_{\Lambda}(\bar{z})
\end{array}\right)+\right. \\
& +\frac{i}{2} \frac{\bar{W}(\bar{z})}{|W|^{2}(z, \bar{z})} \bar{C}_{\overline{i j k}}(z, \bar{z}) g^{i \bar{i}}(z, \bar{z}) g^{j \bar{j}}(z, \bar{z}) g^{k \bar{k}}(z, \bar{z}) \cdot \\
& \left.\cdot\left(D_{j} W\right)(z, \bar{z}, \Gamma)\left(D_{k} W\right)(z, \bar{z}, \Gamma)\left(\begin{array}{c}
\left(D_{i} X^{\Lambda}\right)(z, \bar{z}) \\
\left(D_{i} F_{\Lambda}\right)(z, \bar{z})
\end{array}\right)\right]
\end{aligned}
$$

by simply replacing $\Gamma$ with $\mathcal{H}(\tau)$. On the other hand, the non-BPS $Z \neq 0$ attractor flow solutions obtained in [28] for the $D 2-D 6$ and $D 0-D 2-D 4-D 6$ configurations (both without $B$-fields) consistently do satisfy Eq. (88).

Furthermore, Eq. (89) is the near-horizon $(\tau \rightarrow-\infty)$ limit of Eq. (88), as it has to be.

The issue concerning whether (in all non-BPS $Z \neq 0$-supporting configurations) the actual non-BPS $Z \neq 0$ stabilization Eqs. (if any) admit a $\left(\frac{1}{2}\right.$-)BPS-like reformulation in terms of a non-BPS $Z \neq 0$ fake superpotential (whose general form is given by Eq. (90) below) is open, and its investigation is left for future work.

Next, we can compute the non-BPS $Z \neq 0$ fake superpotential of the first order formalism in the $D 0-D 2-D 4-D 6$ configuration. To do this, we apply the $U$-duality transformation (57-(59) (along with Eqs. (61)-(65)) to the expression of the non-BPS $Z \neq 0$ fake superpotential in the $D 0-D 6$ configuration given by Eq. (74), and, by noticing that $\mathcal{W}$ does not have any further covariance property 
under such a transformation, after some algebra one achieves the following result:

$$
\begin{aligned}
& \mathcal{W}_{\text {non }-B P S, Z \neq 0}\left(\mathcal{Z}, \overline{\mathcal{Z}}, p^{0}, p^{1}, p^{2}, p^{3}, q_{0}, q_{1}, q_{2}, q_{3}\right)= \\
= & \frac{1}{4} \frac{\nu^{3 / 2}\left(-\mathcal{I}_{4}\right)^{1 / 4}}{\sqrt{\prod_{i}\left(\varsigma_{i}+\varrho_{i}\right)}} e^{K / 2}\left[\prod_{i}\left|\varsigma_{i}-\mathcal{Z}^{i}+\left(\varrho_{i}+\mathcal{Z}^{i}\right) e^{-\alpha_{i}} \nu^{-1}\right|\right] \cdot \\
& \cdot\left(1+\sum_{i<j} \frac{\left[\left|\varsigma_{i}-\mathcal{Z}^{i}\right|^{2}-\left|\varrho_{i}+\mathcal{Z}^{i}\right|^{2} e^{-2 \alpha_{i}} \nu^{-2}\right]\left[\left|\varsigma_{j}-\mathcal{Z}^{j}\right|^{2}-\left|\varrho_{j}+\mathcal{Z}^{j}\right|^{2} e^{-2 \alpha_{j}} \nu^{-2}\right]}{\left|\varsigma_{i}-\mathcal{Z}^{i}+\left(\varrho_{i}+\mathcal{Z}^{i}\right) e^{-\alpha_{i}} \nu^{-1}\right|^{2}\left|\varsigma_{j}-\mathcal{Z}^{j}+\left(\varrho_{j}+\mathcal{Z}^{j}\right) e^{-\alpha_{j}} \nu^{-1}\right|^{2}}+\right. \\
& \left.-\sum_{i<j} \frac{e^{-\alpha_{i}-\alpha_{j}} \nu^{-2}\left(\varsigma_{i}+\varrho_{i}\right)\left(\varsigma_{j}+\varrho_{j}\right)\left(\mathcal{Z}^{i}-\overline{\mathcal{Z}}^{\bar{i}}\right)\left(\mathcal{Z}^{j}-\overline{\mathcal{Z}}^{j}\right)}{\left|\mathcal{Z}^{i}+\left(\varrho_{i}+\mathcal{Z}^{i}\right) e^{-\alpha_{i}} \nu^{-1}\right|^{2}\left|\varsigma_{j}-\mathcal{Z}^{j}+\left(\varrho_{j}+\mathcal{Z}^{j}\right) e^{-\alpha_{j}} \nu^{-1}\right|^{2}}\right) .
\end{aligned}
$$

Consistently with the first order formalism [118] for $d=4$ extremal BHs [37, 42] (see also [70] and [74]), it is easy to check that the near-horizon limit of $\mathcal{W}_{\text {non-BPS,Z } \neq 0}^{2}$ yields the square root of $-\mathcal{I}_{4}$ (given by Eq. (8)), or equivalently the square root of the Cayley's hyperdeterminant Det $(\Psi)$ :

$$
\begin{aligned}
& \mathcal{W}_{\text {non-BPS,Z } \neq 0, H}^{2}\left(\Gamma_{D 0-D 2-D 4-D 6}\right) \equiv
\end{aligned}
$$

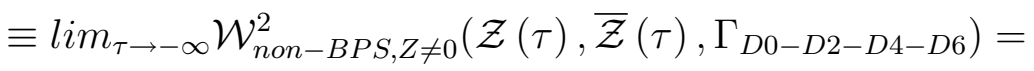

$$
\begin{aligned}
& =\sqrt{-\mathcal{I}_{4}}=\sqrt{\operatorname{Det}(\Psi)}=\frac{S_{B H, n o n-B P Z, Z \neq 0}\left(\Gamma_{D 0-D 2-D 4-D 6}\right)}{\pi},
\end{aligned}
$$

where in the last step the Bekenstein-Hawking entropy-area formula [95] was used.

Now, as done for the $D 0-D 6$ configuration in the previous Subsection, by exploiting the first order formalism [118] for $d=4$ extremal BHs [37, 42] (see also [70] and [74]), one can compute the relevant BH parameters, such as the ADM mass (Eq. (19)) and the covariant scalar charges (Eq. (20)), starting from the fake superpotential $\mathcal{W}_{\text {non-BPS,Z } \neq 0}$ given by Eq. (90). The computations are long but straightforward, and they yield cumbersome results (also e.g. in the limit of " $t^{3}$-degeneration", see Eq. (116) below), which we thus decide to omit here. We will explicitly analyze some particular configurations in Sect. 6.

However, it is easy to realize that Eq. (90) implies the marginal bound [111] to be saturated, because (see Eq. (19))

$$
\begin{aligned}
& M_{A D M, n o n-B P S, Z \neq 0,}\left(\mathcal{Z}_{\infty}, \overline{\mathcal{Z}}_{\infty}, \Gamma_{D 0-D 2-D 4-D 6}\right)=
\end{aligned}
$$

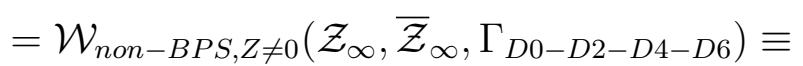

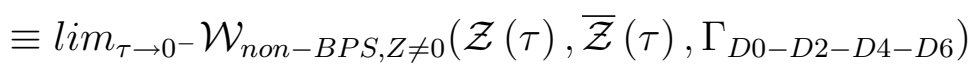

is equal to the sum of the $A D M$ masses of four $D 6$-branes with appropriate fluxes (for further detail on definition of such brane fluxes, see the related discussion in [59]). Thus, generalizing the related results of [59] and [62], it can be stated that the marginal stability holds for the most general non-BPS $Z \neq 0$ attractor flow of the $\mathcal{N}=2, d=4$ stu model.

\section{Analysis of Particular Configurations}

In this Section we analyze in depth some particularly simple configurations, generalizing some results in literature $[28,51,59,62]$. 


\subsection{Magnetic $(D 0-D 4)$}

The configuration $D 0-D 4$ (also named magnetic) of the stu model has been previously treated in [51] and [59]. In this case, the quantities of the $U$-duality transformation (57)-(59) along $\mathcal{O}_{\text {non-BPS,Z } \neq 0}$ defined by Eqs. (62)-(64) undergo a major simplification:

$$
\xi=\xi_{0} ; \quad \varsigma_{i}=\varrho_{i}=\sqrt{\frac{-q_{0} p^{i}}{\frac{1}{2} s_{i j k} p^{j} p^{k}}} .
$$

Correspondingly, the non-BPS $Z \neq 0$ attractor flow (81)-(83) acquires the following form (as above, the moduli are here denoted as $\mathcal{Z}^{i} \equiv \mathcal{X}^{i}-i \mathcal{Y}^{i} ; i \neq j \neq l$ and no sum on repeated $i$-indices throughout):

$$
\begin{aligned}
& \exp \left[-4 U_{n o n-B P S, Z \neq 0}(\tau)\right]=h_{0}(\tau) h_{1}(\tau) h_{2}(\tau) h_{3}(\tau)-b^{2} ; \\
& \mathcal{X}_{n o n-B P S, Z \neq 0}^{i}(\tau)=\varsigma_{i} \cdot \\
& \cdot \frac{e^{2 \alpha_{i}}\left[h_{j}(\tau) h_{l}(\tau)+h_{0}(\tau) h_{i}(\tau)+2 b\right]-\left[h_{j}(\tau) h_{l}(\tau)+h_{0}(\tau) h_{i}(\tau)-2 b\right]}{\left\{\begin{array}{l}
e^{2 \alpha_{i}}\left[h_{j}(\tau) h_{l}(\tau)+h_{0}(\tau) h_{i}(\tau)+2 b\right]+2 e^{\alpha_{i}}\left[h_{j}(\tau) h_{l}(\tau)-h_{0}(\tau) h_{i}(\tau)\right]+ \\
+h_{j}(\tau) h_{l}(\tau)+h_{0}(\tau) h_{i}(\tau)-2 b
\end{array}\right\}} ; \\
& \mathcal{Y}_{n o n-B P S, Z \neq 0}^{i}(\tau)=4 \varsigma_{i}
\end{aligned}
$$

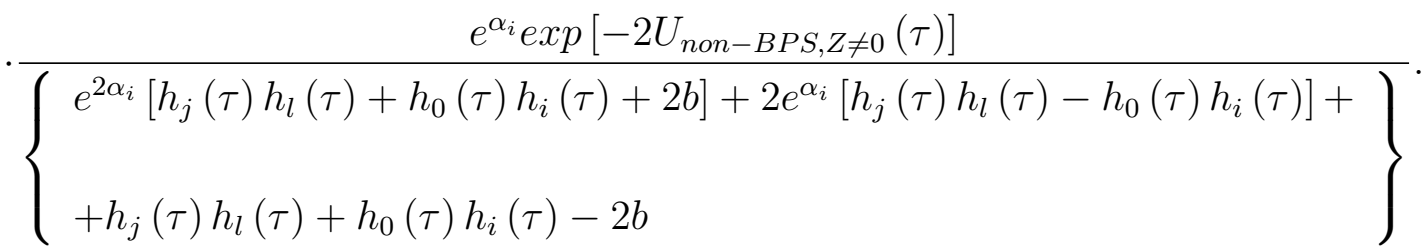

It is worth pointing out that the $D 0-D 4$ configuration supports axion-free non-BPS $Z \neq 0$ attractor flow(s); when considering the near-horizon limit, and thus the critical, charge-dependent values of the moduli, this is consistent with the analysis performed in [15, 41, 47]. An axion-free attractor flow solution of Eqs. (94)-(96) can be obtained e.g. by putting

$$
\begin{aligned}
\alpha_{i} & =0 \forall i=1,2,3 ; \\
b & =0,
\end{aligned}
$$

and it reads as follows:

$$
\begin{aligned}
& \exp \left[-4 U_{\text {non-BPS,Z } \neq 0, \text { axion-free }}(\tau)\right]=h_{0}(\tau) h_{1}(\tau) h_{2}(\tau) h_{3}(\tau) ; \\
& \mathcal{X}_{\text {non-BPS,Z } \neq 0, \text { axion-free }}^{i}(\tau)=0 \text {; } \\
& \mathcal{Y}_{\text {non-BPS,Z } \neq 0, \text { axion-free }}^{i}(\tau)=4 \varsigma_{i} \frac{\sqrt{h_{0}(\tau) h_{1}(\tau) h_{2}(\tau) h_{3}(\tau)}}{4 h_{j}(\tau) h_{l}(\tau)} .
\end{aligned}
$$


Furthermore, within the additional assumption (97), Eqs. (94)-(96) yield the solution obtained in [59].

Always considering a framework in which the assumption (97) holds true, Eqs. (90) yields that the non-BPS $Z \neq 0$ fake superpotential in the $D 0-D 4$ configuration has the following expression:

$$
\begin{gathered}
\left.\mathcal{W}_{\text {non-BPS }, Z \neq 0}\right|_{\alpha_{i}=0 \forall i}\left(\mathcal{Z}, \overline{\mathcal{Z}}, \Gamma_{D 0-D 4}\right)=e^{K / 2} \\
\cdot\left[-q_{0}+\frac{p^{1}}{2}\left(\mathcal{Z}^{2} \overline{\mathcal{Z}}^{3}+\mathcal{Z}^{3} \overline{\mathcal{Z}}^{2}\right)+\frac{p^{2}}{2}\left(\mathcal{Z}^{1} \overline{\mathcal{Z}}^{3}+\mathcal{Z}^{3} \overline{\mathcal{Z}}^{1}\right)+\frac{p^{3}}{2}\left(\mathcal{Z}^{2} \overline{\mathcal{Z}}^{1}+\mathcal{Z}^{1} \overline{\mathcal{Z}}^{2}\right)\right] .
\end{gathered}
$$

The axion-free version of such a fake superpotential (e.g. pertaining to the solution (99)-(99)) reads as follows:

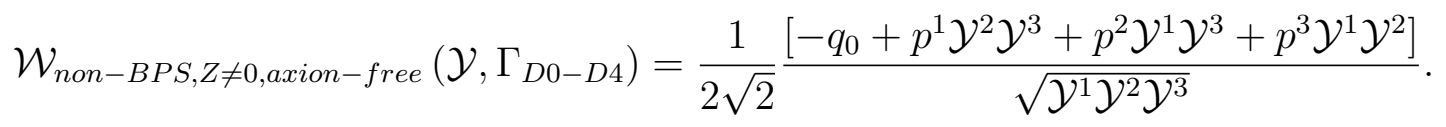

The existence of a first order formalism in the non-BPS $Z \neq 0$-supporting (branch of the) $D 0-D 4$ configuration of the stu model, based on the fake superpotential given by Eq. (100), gives a simple explanation of the integrability of the equations of motion of scalars, answering to the question raised in Appendix A of [59].

Now, as done above for the $D 0-D 6$ and $D 0-D 2-D 4-D 6$ configurations, by exploiting the first order formalism for $d=4$ extremal BHs, one can compute the relevant BH parameters, such as the

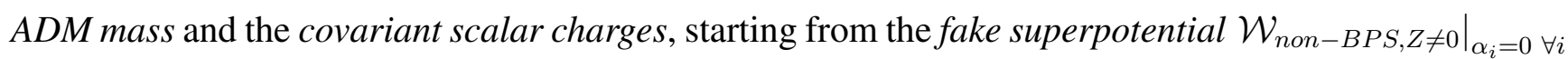
given by Eq. (100).

Concerning the ADM mass, by recalling Eq. (19) and using Eq. (100) one obtains an explicit expression which, after introducing suitable dressed charges (see Eq. (104)) and putting (see Eq. (77))

$$
B^{1}=B^{2}=B^{3}=B,
$$

is given by Eq. (4.6) of [59], which we report here for completeness' sake:

$$
\begin{aligned}
& \left.M_{A D M, \text { non }-B P S, Z \neq 0}\right|_{\alpha_{i}=0 \forall i}\left(\mathcal{Z}_{\infty}, \overline{\mathcal{Z}}_{\infty}, \Gamma_{D 0-D 4}\right)= \\
& =\left.\lim _{\tau \rightarrow 0^{-}} \mathcal{W}_{\text {non-BPS,Z} \neq 0}\right|_{\alpha_{i}=0 \forall i}\left(\mathcal{Z}(\tau), \overline{\mathcal{Z}}(\tau), \Gamma_{D 0-D 4}\right)= \\
& =\frac{1}{2 \sqrt{2}}\left[\left|Q_{0}\right|+\left(1+B^{2}\right) \sum_{i} P^{i}\right],
\end{aligned}
$$

where the dressed charges are defined as follows (no summation on repeated indices; notice the different definitions with respect to the $D 0-D 6$ configuration, whose dressed charges are given by Eq. (77)):

$$
Q_{0} \equiv \frac{q_{0}}{\sqrt{\mathcal{Y}_{\infty}^{1} \mathcal{Y}_{\infty}^{2} \mathcal{Y}_{\infty}^{2}}}, \quad P^{i} \equiv \frac{\sqrt{\mathcal{Y}_{\infty}^{1} \mathcal{Y}_{\infty}^{2} \mathcal{Y}_{\infty}^{2}}}{\mathcal{Y}_{\infty}^{i}} p^{i}
$$

By recalling Eq. (20) and using Eq. (100), one can compute the covariant scalar charges of the non-BPS $Z \neq 0$ attractor flow in the $D 0-D 4$ configuration. Within the simplifying assumptions (97) and (102), one obtains the following explicit expressions $(i \neq j \neq l$, no sum on repeated indices):

$$
\begin{gathered}
\Sigma_{\mathcal{X}, i, n o n-B P S, Z \neq 0}\left(\mathcal{X}_{\infty}, \mathcal{Y}_{\infty}, \Gamma_{D 0-D 4}\right) \equiv \\
\equiv \lim _{\tau \rightarrow 0^{-}}\left(\frac{\left.\partial \mathcal{W}_{n o n-B P S, Z \neq 0}\right|_{\alpha_{m}=0 \forall m}}{\partial \mathcal{X}^{i}}\right)\left(\mathcal{Z}(\tau), \overline{\mathcal{Z}}(\tau), \Gamma_{D 0-D 4}\right)= \\
=\sqrt{2} \mathcal{X}_{\infty}^{i}\left(P^{j}+P^{l}\right)
\end{gathered}
$$




$$
\begin{aligned}
& \Sigma_{\mathcal{Y}, i, n o n-B P S, Z \neq 0}\left(\mathcal{X}_{\infty}, \mathcal{Y}_{\infty}, \Gamma_{D 0-D 4}\right) \equiv \\
& \equiv \lim _{\tau \rightarrow 0^{-}}\left(\frac{\left.\partial \mathcal{W}_{n o n-B P S, Z \neq 0}\right|_{\alpha_{m}=0 \forall m}}{\partial \mathcal{Y}^{i}}\right)\left(\mathcal{Z}(\tau), \overline{\mathcal{Z}}(\tau), \Gamma_{D 0-D 4}\right)= \\
& =\frac{\mathcal{Y}_{\infty}^{i}}{\sqrt{2}}\left(-\left|Q_{0}\right|-2 P^{i}+\left(1-B^{2}\right) \sum_{k} P^{k}\right),
\end{aligned}
$$

where the split in axionic scalar charges $\Sigma_{\mathcal{X}, i}$ and dilatonic scalar charges $\Sigma_{\mathcal{Y}, i}$ was performed.

It is here worth computing the difference between the squared non-BPS $Z \neq 0$ fake superpotential and the squared absolute value of the $\mathcal{N}=2, d=4$ central charge along the non-BPS $Z \neq 0$ attractor flow. This amounts to computing the difference generalizing the BPS bound [112] to the whole attractor flow (in the non-BPS $Z \neq 0$-supporting branch of the magnetic charge configuration):

$$
\Delta(\mathcal{Y}, \Gamma) \equiv \mathcal{W}_{\text {non-BPS,Z } \neq 0}^{2}-|Z|^{2}=\frac{\left|q_{0}\right|}{2}\left(\frac{p^{1}}{\mathcal{Y}^{1}}+\frac{p^{2}}{\mathcal{Y}^{2}}+\frac{p^{3}}{\mathcal{Y}^{3}}\right)>0 .
$$

$\Delta$ is dilaton-dependent and strictly positive all along the non-BPS $Z \neq 0$ attractor flow. At the infinity, by using the dressed charges defined by Eq. (104), the result given by Eq. (4.8) of [59] is recovered. Thus, the BPS bound [112] holds not only at the BH event horizon $\left(r=r_{H}\right)$, but actually (in a dilatondependent way) all along the non-BPS $Z \neq 0$ attractor flow (i.e. $\forall r \geqslant r_{H}$ ).

Of course, by relaxing the simplifying conditions (97) and/or (102), i.e. by considering non-vanishing $\alpha_{i}$ s (constrained by Eq. (71)) and/or different, $i$-indexed $B$-fields, a much richer situation arises, but the main features of the framework, outlined above, are left unchanged.

\section{2. $\quad$ Electric $(D 2-D 6)$}

The configuration $D 2-D 6$ (also named electric) of the stu model has been previously treated in [28] and [62]. Analogously to what happens in the D0-D4 (magnetic) configuration, in this case the quantities of the $U$-duality transformation (57)-(59) along $\mathcal{O}_{\text {non-BPS,Z } \neq 0}$ defined by Eqs. (62)-(64) undergo a major simplification (the prime denotes the charges in the considered configuration):

$$
\xi=-\xi_{0} ; \quad \varsigma_{i}=\varrho_{i}=-\sqrt{\frac{\frac{1}{2} s_{i j k} q_{j}^{\prime} q_{k}^{\prime}}{p^{\prime} q_{i}^{\prime}}} .
$$

Correspondingly, the non-BPS $Z \neq 0$ attractor flow (81)-(83) acquires the following form (as above, 
the moduli are here denoted as $\mathcal{Z}^{i} \equiv \mathcal{X}^{i}-i \mathcal{Y}^{i} ; i \neq j \neq l$ and no sum on repeated $i$-indices throughout):

$$
\begin{aligned}
& \exp \left[-4 U_{n o n-B P S, Z \neq 0}(\tau)\right]=h_{0}(\tau) h_{1}(\tau) h_{2}(\tau) h_{3}(\tau)-b^{2} ; \\
& \mathcal{X}_{n o n-B P S, Z \neq 0}^{i}(\tau)=\varsigma_{i} \cdot \\
& \cdot \frac{e^{2 \alpha_{i}}\left[h_{j}(\tau) h_{l}(\tau)+h_{0}(\tau) h_{i}(\tau)+2 b\right]-\left[h_{j}(\tau) h_{l}(\tau)+h_{0}(\tau) h_{i}(\tau)-2 b\right]}{\left\{\begin{array}{l}
e^{2 \alpha_{i}}\left[h_{j}(\tau) h_{l}(\tau)+h_{0}(\tau) h_{i}(\tau)+2 b\right]-2 e^{\alpha_{i}}\left[h_{j}(\tau) h_{l}(\tau)-h_{0}(\tau) h_{i}(\tau)\right]+ \\
+h_{j}(\tau) h_{l}(\tau)+h_{0}(\tau) h_{i}(\tau)-2 b
\end{array}\right\}} \\
& \mathcal{Y}_{n o n-B P S, Z \neq 0}^{i}(\tau)=-4 \varsigma_{i} \cdot \\
& \cdot \frac{e^{\alpha_{i}} e x p\left[-2 U_{n o n-B P S, Z \neq 0}(\tau)\right]}{\left\{\begin{array}{l}
e^{2 \alpha_{i}}\left[h_{j}(\tau) h_{l}(\tau)+h_{0}(\tau) h_{i}(\tau)+2 b\right]-2 e^{\alpha_{i}}\left[h_{j}(\tau) h_{l}(\tau)-h_{0}(\tau) h_{i}(\tau)\right]+ \\
+h_{j}(\tau) h_{l}(\tau)+h_{0}(\tau) h_{i}(\tau)-2 b
\end{array}\right\}}
\end{aligned}
$$

It is worth pointing out that the $D 2-D 6$ configuration supports axion-free non-BPS $Z \neq 0$ attractor flow(s); when considering the near-horizon limit, and thus the critical, charge-dependent values of the moduli, this is consistent with the analysis performed in [15, 41, 47]. An axion-free attractor flow solution of Eqs. (109)-(111) can be obtained e.g. by assuming the conditions given by Eqs. (97) and (98), and it reads as follows:

$$
\begin{aligned}
& \exp \left[-4 U_{\text {non-BPS,Z } \neq 0, \text { axion-free }}(\tau)\right]=h_{0}(\tau) h_{1}(\tau) h_{2}(\tau) h_{3}(\tau) ;
\end{aligned}
$$

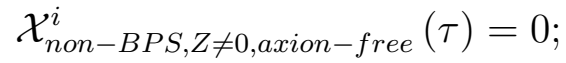

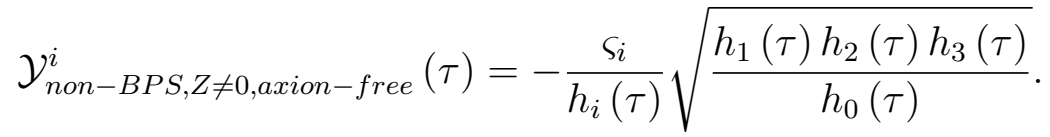

As done for the magnetic configuration, in order to further simplify Eqs. (109)-(111) and (112)-(113), one can consider the particular case constrained by Eq. (97). Within such an additional assumption, the solution obtained in [62], generalizing the one of [28], is recovered.

Furthermore, within the simplifying assumption (97), Eq. (90) yields that the non-BPS $Z \neq 0$ fake superpotential in the $D 2-D 6$ configuration has the following expression:

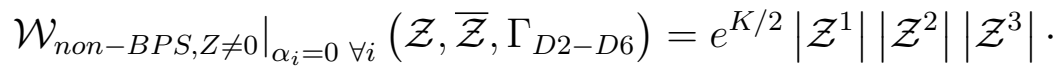

$$
\begin{aligned}
& \cdot\left[p^{\prime 0}+\frac{q_{1}^{\prime}}{2} \frac{\left(\mathcal{Z}^{2} \overline{\mathcal{Z}}^{3}+\mathcal{Z}^{3} \overline{\mathcal{Z}}^{2}\right)}{\left|\mathcal{Z}^{2}\right|^{2}\left|\mathcal{Z}^{3}\right|^{2}}+\frac{q_{2}^{\prime}}{2} \frac{\left(\mathcal{Z}^{1} \overline{\mathcal{Z}}^{3}+\mathcal{Z}^{3} \overline{\mathcal{Z}}^{1}\right)}{\left|\mathcal{Z}^{1}\right|^{2}\left|\mathcal{Z}^{3}\right|^{2}}+\frac{q_{3}^{\prime}}{2} \frac{\left(\mathcal{Z}^{2} \overline{\mathcal{Z}}^{1}+\mathcal{Z}^{1} \overline{\mathcal{Z}}^{2}\right)}{\left|\mathcal{Z}^{1}\right|^{2}\left|\mathcal{Z}^{2}\right|^{2}}\right] .
\end{aligned}
$$


The axion-free version of such a fake superpotential (e.g. pertaining to the solution (112)-(113)) reads as follows:

$$
\left.\mathcal{W}_{\text {non-BPS,Z} \neq 0}\right|_{\alpha_{i}=0 \forall i, \text { axion-free }}\left(\mathcal{Z}, \overline{\mathcal{Z}}, \Gamma_{D 2-D 6}\right)=\frac{1}{2 \sqrt{2}} \sqrt{\mathcal{Y}^{1} \mathcal{Y}^{2} \mathcal{Y}^{3}}\left[p^{\prime 0}+\frac{q_{1}^{\prime}}{\mathcal{Y}^{2} \mathcal{Y}^{3}}+\frac{q_{2}^{\prime}}{\mathcal{Y}^{1} \mathcal{Y}^{3}}+\frac{q_{3}^{\prime}}{\mathcal{Y}^{1} \mathcal{Y}^{2}}\right]
$$

coinciding with the fake superpotential given by Eq. (4-20) of [37].

The existence of a first order formalism in the non-BPS $Z \neq 0$-supporting (branch of the) $D 2-D 6$ configuration of the stu model, based on the fake superpotential given by Eq. (114), gives a explanation of the integrability of the equations of motion of scalars supported by the electric configuration (see the treatment of [62]).

Let us now consider the " $t^{3}$-degeneration" of the stu model, in which all charges and moduli are equal, insensitive to $i$-index; in the considered configuration this amounts to putting

$$
\mathcal{Z}^{1}=\mathcal{Z}^{2}=\mathcal{Z}^{3}=\mathcal{Z}, \quad q_{1}^{\prime}=q_{2}^{\prime}=q_{3}^{\prime}=q^{\prime} / 3
$$

(see the treatment in Sect. 5 of [50]). By doing so, Eq. (114) yields the non-BPS $Z \neq 0$ fake superpotential given by Eq. (5.5) of [37], which we report here for completeness' sake:

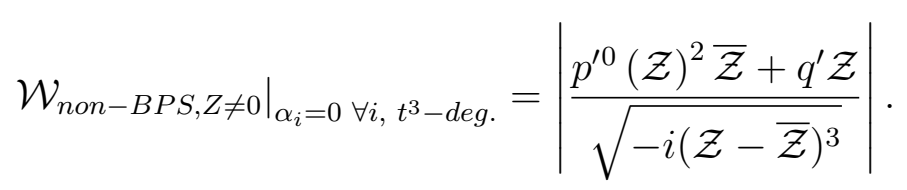

The axion-free version of such a fake superpotential (e.g. pertaining to " $t^{3}$-degeneration" of the solution (112)-(113)) reads as follows:

$$
\mathcal{W}_{\text {non-BPS,Z } \neq 0, \text { axion-free, } t^{3}-\text { deg. }}=\frac{1}{2 \sqrt{2}}\left|\frac{p^{\prime} \mathcal{Y}^{3}+q^{\prime} \mathcal{Y}}{\sqrt{\mathcal{Y}^{3}}}\right|
$$

Now, as done above for the $D 0-D 6, D 0-D 2-D 4-D 6$ and $D 0-D 4$ configurations, by exploiting the first order formalism for $d=4$ extremal BHs, one can compute the relevant BH parameters, such as the ADM mass and the covariant scalar charges, starting from the fake superpotential

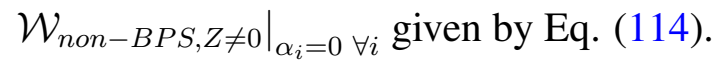

Concerning the ADM mass, by recalling Eq. (19) and using Eqs. (114), (77) and (102), one obtains an explicit expression which, after introducing suitable dressed charges (see Eq. (120)), reads as follows:

$$
\begin{aligned}
& \left.M_{A D M, n o n-B P S, Z \neq 0}\right|_{\alpha_{i}=0 \forall i}\left(\mathcal{Z}_{\infty}, \overline{\mathcal{Z}}_{\infty}, \Gamma_{D 2-D 6}\right)=
\end{aligned}
$$

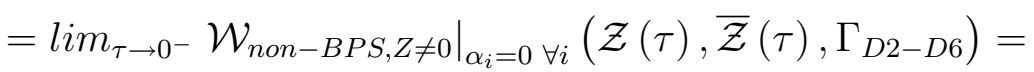

$$
\begin{aligned}
& =\frac{\sqrt{1+B^{2}}}{2 \sqrt{2}}\left[\left(1+B^{2}\right) P^{\prime 0}+\sum_{i} Q_{i}^{\prime}\right] \text {, }
\end{aligned}
$$

where the dressed charges are defined as follows (no summation on repeated indices; notice the different definitions with respect to the $D 0-D 6$ and $D 0-D 4$ configurations, whose dressed charges are given by Eqs. (77) and (104), respectively):

$$
P^{\prime 0} \equiv p^{\prime 0} \sqrt{\mathcal{Y}_{\infty}^{1} \mathcal{Y}_{\infty}^{2} \mathcal{Y}_{\infty}^{2}}, \quad Q_{i}^{\prime} \equiv \frac{\mathcal{Y}_{\infty}^{i}}{\sqrt{\mathcal{Y}_{\infty}^{1} \mathcal{Y}_{\infty}^{2} \mathcal{Y}_{\infty}^{2}}} q_{i}^{\prime} .
$$


Up to redefinition of the dressed charges, Eq. (119) is nothing but Eq. (5.2) of [62].

By recalling Eq. (20) and using Eq. (114), one can compute the covariant scalar charges of the non-BPS $Z \neq 0$ attractor flow in the $D 2-D 6$ configuration. Within the simplifying assumptions (97) and (102), one obtains the following explicit expressions (no sum on repeated indices):

$$
\begin{gathered}
\Sigma_{\mathcal{X}, i, n o n-B P S, Z \neq 0}\left(\mathcal{X}_{\infty}, \mathcal{Y}_{\infty}, \Gamma_{D 2-D 6}\right) \equiv \\
\equiv \lim _{\tau \rightarrow 0^{-}}\left(\frac{\left.\partial \mathcal{W}_{n o n-B P S, Z \neq 0}\right|_{\alpha_{m}=0 \forall m}}{\partial \mathcal{X}^{i}}\right)\left(\mathcal{Z}(\tau), \overline{\mathcal{Z}}(\tau), \Gamma_{D 2-D 6}\right)= \\
=\sqrt{2} \frac{\mathcal{X}_{\infty}^{i}}{\sqrt{1+B^{2}}}\left[\left(1+B^{2}\right) P^{\prime 0}+Q_{i}^{\prime}\right] ; \\
\equiv \lim _{\tau \rightarrow 0^{-}}\left(\frac{\left.\partial \mathcal{W}_{n o n-B P S, Z \neq 0}\right|_{\alpha_{m}=0 \forall m}}{\partial \mathcal{Y}^{i}}\right)\left(\mathcal{Z}(\tau), \overline{\mathcal{Z}}(\tau), \Gamma_{D 2-D 6}\right)= \\
=\frac{\Sigma_{\mathcal{Y}, i, n o n-B P S, Z \neq 0}\left(\mathcal{X}_{\infty}, \mathcal{Y}_{\infty}, \Gamma_{D 2-D 6}\right) \equiv}{\sqrt{2} \sqrt{1+B^{2}}}\left[\left(B^{4}-1\right) P^{\prime 0}-2 Q_{i}^{\prime}+\left(1+B^{2}\right)^{2} \sum_{j} Q_{j}^{\prime}\right]
\end{gathered}
$$

where, as for the $D 0-D 4$ configuration, the split in axionic scalar charges $\Sigma_{\mathcal{X}, i}$ and dilatonic scalar charges $\Sigma_{\mathcal{Y}, i}$ was performed.

As done for the magnetic configuration in Subsect. 6.1., also for electric configuration it is worth computing the difference between the squared non-BPS $Z \neq 0$ fake superpotential and the squared absolute value of the $\mathcal{N}=2, d=4$ central charge along the non-BPS $Z \neq 0$ attractor flow:

$\Delta(\mathcal{X}, \mathcal{Y}, \Gamma) \equiv \mathcal{W}_{n o n-B P S, Z \neq 0}^{2}-|Z|^{2}=\frac{p^{\prime 0}}{2}\left(q_{1}^{\prime} \frac{\left(\mathcal{X}^{1}\right)^{2}+\left(\mathcal{Y}^{1}\right)^{2}}{\mathcal{Y}^{1}}+q_{2}^{\prime} \frac{\left(\mathcal{X}^{2}\right)^{2}+\left(\mathcal{Y}^{2}\right)^{2}}{\mathcal{Y}^{2}}+q_{3}^{\prime} \frac{\left(\mathcal{X}^{3}\right)^{2}+\left(\mathcal{Y}^{3}\right)^{2}}{\mathcal{Y}^{3}}\right)>0$

Differently from what happens for the magnetic configuration, for electric configuration $\Delta$ does depend also on axions, but nevertheless it is still strictly positive all along the non-BPS $Z \neq 0$ attractor flow. At infinity, by using the dressed charges defined by Eq. (120), the following result is achieved:

$$
\Delta\left(\mathcal{X}_{\infty}, \mathcal{Y}_{\infty}, \Gamma\right)=\frac{P^{\prime 0}}{2}\left(1+B^{2}\right) \sum_{i} Q_{i}^{\prime}
$$

Thus, the BPS bound [112] holds not only at the BH event horizon $\left(r=r_{H}\right)$, but actually (in a scalardependent way) all along the non-BPS $Z \neq 0$ attractor flow (i.e. $\forall r \geqslant r_{H}$ ).

Of course, by relaxing the simplifying conditions (97) and/or (102), i.e. by considering non-vanishing $\alpha_{i}$ s (constrained by Eq. (71)) and/or different, $i$-indexed $B$-fields, a much richer situation arises, but the main features of the framework, outlined above, are left unchanged.

By noticing that the D0-D4 (magnetic) and D2-D6 (electric) configurations are reciprocally dual in $d=4$ and recalling the treatment of Subsect. 5.1., it is worth computing the matrices $M_{i, D 0-D 4 \longrightarrow D 2-D 6}$ representing the $U$-duality transformation along the charge orbit $\mathcal{O}_{n o n-B P S, Z \neq 0}$ which connects (the nonBPS $Z \neq 0$-supporting branches of) such two charge configurations. In order to do this, we exploit the treatment given in Subsect. 5.1., by performing the following steps:

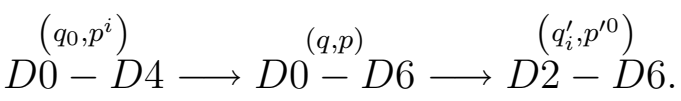


For the step $D 0-D 4 \longrightarrow D 0-D 6$, we consider $M_{i}^{-1}$ given by Eq. (61), along with the definitions (62)-(64) specified for the configuration $D 0-D 4$, obtaining $M_{i, D 0-D 4 \longrightarrow D 0-D 6}^{-1}$. Thence, for the the step $D 0-D 6 \longrightarrow D 2-D 6$, we take $M_{i}$ given by Eq. (61), along with the definitions (62)-(64) specified for the configuration $D 2-D 6$, obtaining $M_{i, D 0-D 6} \longrightarrow D 2-D 6$. Thus (no sum on repeated index $i=1,2,3$ throughout; also recall Eq. (57)):

$$
\begin{aligned}
\left(M_{i, D 0-D 4 \longrightarrow D 2-D 6}\right)_{a^{\prime}}^{b^{\prime}} & =\left(M_{i, D 0-D 6 \longrightarrow D 2-D 6}\right)_{a^{\prime}}^{a}\left(M_{i, D 0-D 4 \longrightarrow D 0-D 6}^{-1}\right)_{a}^{b^{\prime}}= \\
& =\left(\begin{array}{cc}
0 & -\sqrt[4]{-\frac{q_{0} p^{i} s_{i j k} q_{j}^{\prime} q_{k}^{\prime}}{p^{\prime 0} q_{i}^{\prime} s_{i j k} p^{j} p^{k}}} \\
\sqrt[4]{-\frac{p^{\prime 0} q_{i}^{\prime} s_{i j k} p^{j} p^{k}}{q_{0} p^{i} s_{i j k} q_{j}^{\prime} q_{k}^{\prime}}} & 0
\end{array}\right) .
\end{aligned}
$$

Consequently, by recalling Eqs. (59) and (65) one can directly relate the non-BPS $Z \neq 0$ attractor flows

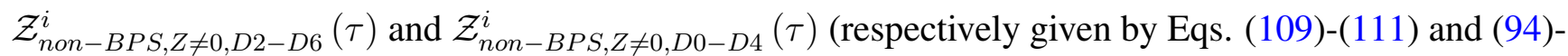
(96); recall that $\mathcal{Z}^{i}(\tau)=\mathcal{X}^{i}(\tau)-i \mathcal{Y}^{i}(\tau)$ ) by the following expression, explicitly showing the duality between the D0 - D4 (magnetic) and D2-D6 (electric) configurations in $d=4$ :

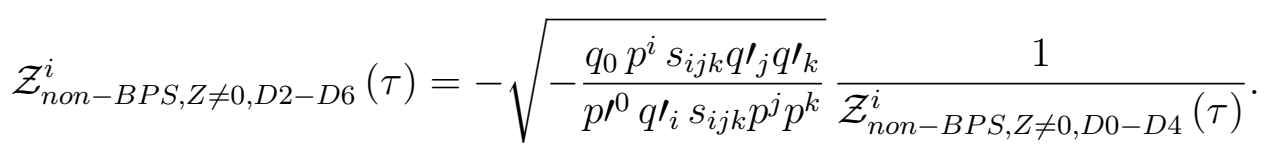

\section{3. $D 0-D 2-D 4$}

The configuration $D 0-D 2-D 4$ of the stu model has been previously treated in [62]. In this case,

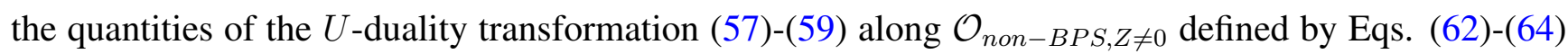
have the following form (no summation on repeated index $i=1,2,3$ throughout):

$$
\xi=\xi_{0} ; \quad \varsigma_{i}=\frac{\sqrt{-\mathcal{I}_{4}}+p^{l} q_{l}-2 p^{i} q_{i}}{s_{i j k} p^{j} p^{k}} ; \quad \varrho_{i}=\frac{\sqrt{-\mathcal{I}_{4}}-p^{l} q_{l}+2 p^{i} q_{i}}{s_{i j k} p^{j} p^{k}} .
$$

Within the additional assumption (97) (considered for simplicity' sake), the non-BPS $Z \neq 0$ attractor flow (81)-(83) correspondingly acquires the following form (as above, the moduli are here denoted as $\mathcal{Z}^{i} \equiv \mathcal{X}^{i}-i \mathcal{Y}^{i} ; i \neq j \neq l$, and no sum on repeated $i$-indices throughout):

$$
\begin{aligned}
& \exp \left[-4 U_{n o n-B P S, Z \neq 0}(\tau)\right]=h_{0}(\tau) h_{1}(\tau) h_{2}(\tau) h_{3}(\tau)-b^{2} \\
& \mathcal{X}_{n o n-B P S, Z \neq 0}^{i}(\tau)=\frac{\sqrt{-\mathcal{I}_{4}}}{s_{i k m} p^{k} p^{m}} \frac{b}{h_{j}(\tau) h_{l}(\tau)}+\frac{p^{n} q_{n}-2 p^{i} q_{i}}{s_{i k m} p^{k} p^{m}} \\
& \mathcal{Y}_{n o n-B P S, Z \neq 0}^{i}(\tau)=\frac{\sqrt{-\mathcal{I}_{4}}}{s_{i k m} p^{k} p^{m}} \frac{\exp \left[-2 U_{n o n-B P S, Z \neq 0}(\tau)\right]}{h_{j}(\tau) h_{l}(\tau)}
\end{aligned}
$$

This is nothing but the solution obtained in [62].

It is worth pointing out that, as the general case $D 0-D 2-D 4-D 6$ (see Subsect. 5.3.), the $D 0-D 2-D 4$ configuration does not support axion-free non-BPS $Z \neq 0$ attractor flow(s); when considering the near-horizon limit, and thus the critical, charge-dependent values of the moduli, this is consistent with the analysis performed in [15, 41, 47]. 
Furthermore, always within the simplifying assumption (97), Eq. (90) yields that the non-BPS $Z \neq 0$ fake superpotential in the $D 0-D 2-D 4$ configuration has the following expression:

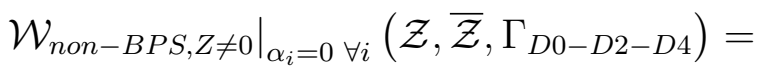

$$
\begin{aligned}
& =e^{K / 2}\left[-q_{0}-\frac{q_{1}}{2}\left(\mathcal{Z}^{1}+\overline{\mathcal{Z}}^{1}\right)-\frac{q_{2}}{2}\left(\mathcal{Z}^{2}+\overline{\mathcal{Z}}^{2}\right)-\frac{q_{3}}{2}\left(\mathcal{Z}^{3}+\overline{\mathcal{Z}}^{3}\right)+\right. \\
& \left.+\frac{p^{1}}{2}\left(\mathcal{Z}^{2} \overline{\mathcal{Z}}^{3}+\mathcal{Z}^{3} \overline{\mathcal{Z}}^{2}\right)+\frac{p^{2}}{2}\left(\mathcal{Z}^{1} \overline{\mathcal{Z}}^{3}+\mathcal{Z}^{3} \overline{\mathcal{Z}}^{1}\right)+\frac{p^{3}}{2}\left(\mathcal{Z}^{1} \overline{\mathcal{Z}}^{2}+\mathcal{Z}^{2} \overline{\mathcal{Z}}^{1}\right)\right] \text {. }
\end{aligned}
$$

The existence of a first order formalism in the non-BPS $Z \neq 0$-supporting (branch of the) $D 0-D 2-D 4$ configuration of the stu model, based on the fake superpotential given by Eq. (132), gives a explanation of the integrability of the equations of motion of scalars supported by such a configuration (see the treatment of [62]).

Now, by exploiting the first order formalism for $d=4$ extremal BHs, one can compute the relevant $\mathrm{BH}$ parameters, such as the ADM mass and the covariant scalar charges, starting from the fake superpotential $\left.\mathcal{W}_{\text {non-BPS,Z } \neq 0}\right|_{\alpha_{i}=0 \forall i}$ given by Eq. (132).

Concerning the ADM mass, by recalling Eq. (19) and using Eq. (132) one obtains the following result:

$$
\begin{aligned}
& \left.M_{A D M, n o n-B P S, Z \neq 0}\right|_{\alpha_{i}=0 \forall i}\left(\mathcal{Z}_{\infty}, \overline{\mathcal{Z}}_{\infty}, \Gamma_{D 0-D 2-D 4}\right)=
\end{aligned}
$$

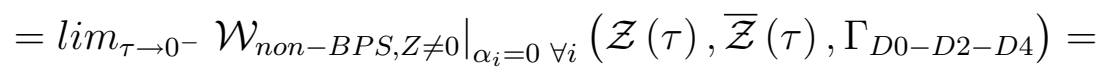

$$
\begin{aligned}
& =\frac{1}{2 \sqrt{2}}\left[\left|Q_{0}\right|-\sum_{i} Q_{i} B_{i}+\sum_{i} P^{i}+\sum_{i \neq j \neq k} P^{i} B_{j} B_{k}\right] \text {, }
\end{aligned}
$$

where the dressed charges are defined by Eqs. (104) and (120).

By recalling Eq. (20) and using Eq. (132), one can compute the covariant scalar charges of the non-BPS $Z \neq 0$ attractor flow in the $D 0-D 2-D 4$ configuration. Always within the assumption (97), one obtains the following explicit expressions (no sum on repeated index $i=1,2,3$ ):

$$
\begin{aligned}
& \Sigma_{\mathcal{X}, i, n o n-B P S, Z \neq 0}\left(\mathcal{X}_{\infty}, \mathcal{Y}_{\infty}, \Gamma_{D 0-D 2-D 4}\right) \equiv \\
& \equiv \lim _{\tau \rightarrow 0^{-}}\left(\frac{\left.\partial \mathcal{W}_{n o n-B P S, Z \neq 0}\right|_{\alpha_{m}=0 \forall m}}{\partial \mathcal{X}^{i}}\right)\left(\mathcal{Z}(\tau), \overline{\mathcal{Z}}(\tau), \Gamma_{D 0-D 2-D 4}\right)= \\
& =\sqrt{2} \mathcal{Y}_{\infty}^{i}\left(s_{i j k} P^{j} B_{k}-Q_{i}\right) \text {; } \\
& \Sigma_{\mathcal{Y}, i, n o n-B P S, Z \neq 0}\left(\mathcal{X}_{\infty}, \mathcal{Y}_{\infty}, \Gamma_{D 0-D 2-D 4}\right) \equiv
\end{aligned}
$$

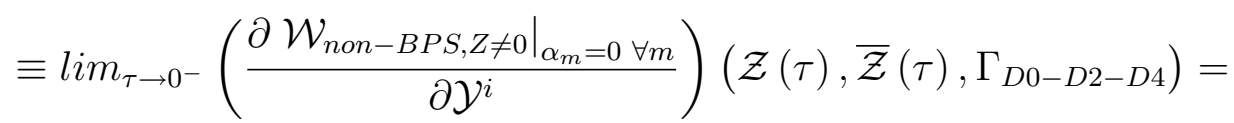

$$
\begin{aligned}
& =\frac{\mathcal{Y}_{\infty}^{i}}{\sqrt{2}}\left[-\left|Q_{0}\right|-2 P^{i}+\sum_{j} Q_{j} B_{j}+\sum_{j} P^{j}-\sum_{i \neq j \neq k} P^{i} B_{j} B_{k}\right],
\end{aligned}
$$

where, as above, the split in axionic scalar charges $\Sigma_{\mathcal{X}, i}$ and dilatonic scalar charges $\Sigma_{\mathcal{Y}, i}$ was performed, and the definition (77) of B-fields was used.

As done for the magnetic and electric configurations (respectively in Subsects. 6.1. and 6.2.), also for $D 0-D 2-D 4$ configuration it is worth computing the difference between the squared non-BPS 
$Z \neq 0$ fake superpotential and the squared absolute value of the $\mathcal{N}=2, d=4$ central charge along the non-BPS $Z \neq 0$ attractor flow. For simplicity's sake, we decide to perform computations in the " $t^{3}$-degeneration" of the stu model, in which all charges and moduli are equal, insensitive to $i$-index; in the considered configuration this amounts to putting (see the treatment in Sect. 5 of [50])

$$
\mathcal{Z}^{1}=\mathcal{Z}^{2}=\mathcal{Z}^{3}=\mathcal{Z} \equiv \mathcal{X}-i \mathcal{Y}, q_{1}=q_{2}=q_{3}=q / 3, p^{1}=p^{2}=p^{3}=p,
$$

which generalizes Eq. (116), also implying the assumption (102). Then, one obtains the following result:

$$
\Delta(\mathcal{Y}, \Gamma) \equiv \mathcal{W}_{\text {non-BPS,Z } \neq 0}^{2}-|Z|^{2}=\frac{1}{8 \mathcal{Y}}\left(12\left|q_{0}\right| p-q^{2}\right)>0
$$

In this case, $\Delta$ is strictly positive all along the non-BPS $Z \neq 0$ attractor flow, due to the fact that $\mathcal{I}_{4}$ is strictly negative. At infinity, by using the dressed charges defined by Eqs. (104) and (120), the following result is achieved:

$$
\Delta\left(\mathcal{Y}_{\infty}, \Gamma\right)=\frac{1}{8}\left(12\left|Q_{0}\right| P-Q^{2}\right)
$$

Thus, the BPS bound [112] is found to hold not only at the BH event horizon $\left(r=r_{H}\right)$, but actually (in a scalar-dependent way) all along the non-BPS $Z \neq 0$ attractor flow (i.e. $\forall r \geqslant r_{H}$ ).

It is here worth pointing out that, by exploiting the procedure outlined in Subsect. 5.1., the results (107), (123) and (137) can be related one to the others by performing suitable $U$-duality transformations. In such a way (or equivalently by recalling the results of Sect. 2. and Subsect. 5.3.), one can also compute $\Delta$ for the non-BPS $Z \neq 0$-supporting branch of the most general (i.e. $D 0-D 2-D 4-D 6$ ) $\mathrm{BH}$ charge configuration.

Of course, by relaxing the simplifying condition (97) and/or the " $t^{3}$-degeneration" described by Eq. (136) (in turn implying the condition(102)), a much richer situation arises, but the main features of the framework, outlined above, are left unchanged.

\section{Conclusion}

In the present paper the analysis and solution of the equations of motion of the scalar fields of the socalled stu model [15, 39, 41, 50,97-100], consisting of $\mathcal{N}=2, d=4$ ungauged supergravity coupled to 3 Abelian vector multiplets whose complex scalars span the special Kähler manifold $\frac{G}{H}=\left(\frac{S U(1,1)}{U(1)}\right)^{3}$, has been performed in full detail. The obtained results complete and generalize the ones already present in literature [28, 51, 59, 62].

The 3 classes of non-degenerate attractor flows of the stu model have been presented and/or determined in full generality, and their features have been studied and compared. We sketchily list the essential facts below:

- The $\frac{1}{2}$-BPS attractor flow, known since [105]- [109] (as well as the third of Refs. [111]), has been reviewed. It corresponds to $\mathcal{I}_{4}>0$, and it does not yield any associated moduli space, at the BH event horizon nor along the flow. The most general flow solution (9) can be obtained starting from the known most general horizon, critical solution [15, 28, 39, 41, 50, 99, 100], and replacing the $\mathrm{BH}$ charges with suitable harmonic functions. Correspondingly, the $\frac{1}{2}$-BPS Attractor Eqs. (15), determining the attractor solution at BH event horizon, can be extended to the whole 
flow into the so-called $\frac{1}{2}$-BPS stabilization Eqs. (14) by simply substituting the BH charges with the corresponding harmonic functions in the radial parameter $\tau$. The (first order) $\frac{1}{2}$-BPS fake superpotential (which is nothing but the absolute value of the $\mathcal{N}=2, d=4$ central charge function $Z$ given by Eq. (5), and thus it is manifesly $H$-invariant) has been explicitly determined, and the relevant BH parameters, namely the gravitational ADM mass (22) and (covariant) scalar charges, have been computed, as functions of BH charges and (spatially) asymptotical scalars. The marginal bound has been shown to be never saturated, and thus marginal stability [111] does not hold for $\frac{1}{2}$-BPS states in the considered framework.

- The non-BPS $Z=0$ attractor flow, hitherto unknown (up to a short comment in [59]), has been studied and derived in full generality. As the $\frac{1}{2}$-BPS attractor flow, it corresponds to $\mathcal{I}_{4}>0$, and it does not yield any associated moduli space, at the BH event horizon nor along the flow. Due to the underlying triality symmetry of the stu model, 3 different "polarizations" of the results are possible, and choosing one of them does not imply any loss of generality. The most general flow solution (29) can be obtained starting from the known most general horizon, critical solution [50], and replacing the $\mathrm{BH}$ charges with suitable harmonic functions. Correspondingly, the nonBPS $Z=0$ Attractor Eqs. (42), determining the attractor solution at BH event horizon, can be extended to the whole flow into the so-called non-BPS $Z=0$ stabilization Eqs. (41) by simply substituting the $\mathrm{BH}$ charges with the corresponding harmonic functions in the radial parameter $\tau$. The (first order) non-BPS $Z=0$ fake superpotential has been explicitly determined, and it is given by the manifestly $H$-invariant Eqs. (31) and (36) (or equivalently, by Eqs. (34) and (38), or (35) and (37)). The relevant BH parameters, namely the gravitational ADM mass (49) and (covariant) scalar charges, have been computed, as functions of $\mathrm{BH}$ charges and (spatially) asymptotical scalars. Furthermore, the BPS bound [112] has been shown to hold all along the non-BPS $Z=0$ attractor flow (i.e. $\forall r \geqslant r_{H}$ ), and not only at the BH event horizon $\left(r=r_{H}\right)$. The marginal bound has been shown to be never saturated, and thus marginal stability [111] does not hold for nonBPS $Z=0$ states in the considered framework. The strict similarity between $\frac{1}{2} \mathrm{BPS}$ and non-BPS $Z=0$ attractor flows can be explained by noticing that both such flows can be uplifted to the same $\frac{1}{8}$-BPS non-degenerate attractor flow of $\mathcal{N}=8, d=4$ supergravity (see $e$.g. the discussion in [50]).

- The non-BPS $Z \neq 0$ attractor flow, studied in [28, 51, 59, 62] in various configurations, has been here studied and analyzed in full generality. It corresponds to $\mathcal{I}_{4}<0$, and it yields an associated moduli space $(S O(1,1))^{2}$ (which is nothing but the scalar manifold of the $d=5$ uplift of the stu model), both at the BH event horizon [41, 43] and along the flow [59]. Consistently with the analysis performed in [51] for the D0 - D4 (magnetic) configuration, the most general flow solution (81)-(83), supported by the (relevant branch of the) $D 0-D 2-D 4-D 6$ configuration (with all charges switched on), cannot be obtained starting from the known most general horizon, critical solution, and replacing the $\mathrm{BH}$ charges with suitable harmonic functions. The opposite claim of [28] for the $D 2-D 6$ (electric) and $D 0-D 2-D 4-D 6$ configurations is actually due to the fact that (some) B-fields were chosen to vanish therein. Correspondingly, the non-BPS $Z \neq 0$ Attractor Eqs. (89), determining the attractor solution at BH event horizon, cannot be extended to the 
whole flow into the so-called non-BPS $Z \neq 0$ stabilization Eqs. (whose would-be version is given by Eq. (88)) by simply substituting the $\mathrm{BH}$ charges with the corresponding harmonic functions in the radial parameter $\tau$. The (first order) non-BPS $Z \neq 0$ fake superpotential has been explicitly determined, and it is given by the manifestly non- $H$-invariant Eq. (90), which reproduces the few known results [37] in the corresponding particular cases. The relevant BH parameters, namely the gravitational ADM mass and (covariant) scalar charges, have been computed in Subsect. 5.2. and Sect. 6. for various configurations, as functions of BH charges and (spatially) asymptotical scalars. Furthermore, the BPS bound [112] has been shown to hold all along the non-BPS $Z \neq 0$ attractor flow (i.e. $\forall r \geqslant r_{H}$ ), and not only at the BH event horizon $\left(r=r_{H}\right)$. This has been explicitly computed for non-BPS $Z \neq 0$-supporting branches of magnetic (Subsect. 6.1.), electric (Subsect. 6.2.) and $D 0-D 2-D 4$ (Subsect. 6.3.) configurations. On the other hand, by exploiting the procedure outlined in Subsect. 5.1., or equivalently by recalling the results of Sect. 2. and Subsect. 5.3., one can also prove the BPS bound to hold for the non-BPS $Z \neq 0$-supporting branch of the most general (i.e. $D 0-D 2-D 4-D 6$ ) BH charge configuration. From the very Eq. (90), the marginal bound turns out to be saturated, and thus marginal stability [111] holds for non-BPS $Z \neq 0$ states in the considered framework. The manifest non- $H$-invariance of the general (first order) non-BPS $Z \neq 0$ fake superpotential (90) seems clash with the $H$-invariance imposed by Eq. (2.21) of [37] (or equivalently by Eq. (13) of [42]), reported as eq. (39) above, relating the fake superpotential and the warp factor $U(\tau)$ appearing in the Ansatz (40)for the static, spherically symmetric, asymptotically flat, extremal dyonic BH metric. A way out to such an apparent contradiction consists in recalling the treatment of Subsect. 2.2. of [37], and thus observing that the fake superpotential is not unique within the same attractor flow, the various equivalent superpotentials being related through a matrix $R$ satisfying the conditions (2.28) and (2.29) of [37] (see in general Subsect. 2.2 of [37]). If $R$ is scalar-dependent, it may relate (manifestly) $H$-invariant fake superpotentials to (manifestly) non- $H$-invariant ones, and vice versa. Thus, one may state that a suitable scalar-dependent matrix $R$ exists, satisfying Eqs. (2.28) and (2.29) of [37], and mapping the non-BPS $Z \neq 0$ fake superpotential (90) into an equivalent, but (manifestly) $H$-invariant one. It would be interesting to determine explicitly such a matrix; we leave such an issue for future work.

Various comments, remarks, ideas for further developments along the lines of research considered in the present paper are listed below.

- By exploiting the approach considered in Sect. 5 of [50], the stu can be consistently related to the so-called $s t^{2}$ and $t^{3}$ models, respectively with 2 and 1 complex scalars. Through such a procedure, all the results obtained for the stu model can be considered to hold for such models. Furthermore, by performing the near-horizon (i.e. $\tau \rightarrow-\infty$ ) limit on the attractor flow solutions, one obtains the corresponding attractor solution at the event horizon of the extremal $\mathrm{BH}$. This is particularly relevant for the non-BPS $Z \neq 0$ horizon attractor solutions, hitherto analytically known (in a rather intricate form) only for the $t^{3}$ model, so far the only $\mathcal{N}=2, d=4$ supergravity model based on cubic special Kähler geometry whose Attractor Eqs. had been completely solved. In the nearhorizon limit, the results of the present paper yield the non-BPS $Z \neq 0$ horizon attractor solutions 
for both $s t^{2}$ and $s t u$ models.

- It should be recalled once again that the stu model is a sector of all $\mathcal{N}>2, d=4$ supergravities, as well as of all $\mathcal{N}=2, d=4$ supergravities based on homogeneous (both symmetric [97, 113115] and non-symmetric - see e.g. [116, 117] -) scalar manifolds based on cubic geometries. Thus, stu model captures the essential features of extremal BHs in all such theories (see e.g. the stu interpretation of $\mathcal{N}=8, d=4$ attractors, and the observations in [59]). Consequently, (the core of) the results holding for stu model can be thought to hold at least for all such theories. For instance, it would be interesting to try to extend them to some of the theories considered in [74], also in relation to the issue of the effective horizon radius treated therein.

- The stu model has been recently shown to be relevant for the analogy between pure states of multipartite entanglement of qubits in quantum information theory and extremal stringy BHs [3] (see also [120] for further recent developments). In the seventh of Refs. [3] the relation between quantum information theory and the theory of extremal stringy BHs was studied within the stu model, showing that the three-qubit interpretation of supersymmetric, $\frac{1}{2}$-BPS attractors can be extended also to include non-supersymmetric, non-BPS (both $Z \neq 0$ and $Z=0$ ) ones, performing a classification of the attractor solutions based on the charge codes of quantum error correction. However, only double-extremal solutions, with constant, non-dynamical scalars all along the attractor flow, were discussed therein. Thus, as also observed in [62], it would be interesting to extend the analysis of the seventh of Refs. [3] using the full general non-BPS (both $Z \neq 0$ and $Z=0$ ) attractor flow solutions obtained in the present paper.

- The existence of a first order formalism for the equations of motion of the scalar fields (also named attractor flow Eqs.) in the background of an extremal BH [37, 42] in principle implies the integrability of such equations, regardless their eventual intricate form. This answers to the question raised in Appendix A of [59], and it is particularly relevant for the non-BPS $Z \neq 0$ attractor flow, as pointed out in Subsects. 6.1.-6.3.. It would be interesting to study the integrability of the equations of motion of the scalars in presence of quantum (perturbative and/or non-perturbative) corrections to the considered stu model. For instance, it would be interesting to study the attractor flow Eqs. for a quantum corrected prepotential $f=s t u+i \lambda$, with $\lambda \in \mathbb{R}$, which is the only correction which preserves the axion shift symmetry and modifies the geometry of the scalar manifold (see [58] and Refs. therein). A tempting ideas, inspired by the intriguing connection between quantum information theory and extremal BHs mentioned at the previous point, is to consider the quantum, axion-shift-consistent parameter $\lambda$ as related to the quantum noise of the system (see e.g. [123] and Refs. therein).

- As found in [121], observed also in [59] and noticed in Sect. 3., an immediate consequence of the general form of $\frac{1}{2}$-BPS attractor flow given by Eq. (9) is that $\Gamma_{\infty}$ satisfies the $\frac{1}{2}$-BPS Attractor Eqs. [106]. This determines a sort of "Attractor Mechanism at spatial infinity", mapping the 6 real moduli $\left(x^{1}, x^{2}, x^{3}, y^{1}, y^{2}, y^{3}\right)$ into the 8 real constants $\left(p_{\infty}^{1}, p_{\infty}^{2}, p_{\infty}^{3}, q_{1, \infty}, q_{2, \infty}, q_{3, \infty}\right)$, arranged as $\Gamma_{\infty}$ and constrained by the 2 real conditions (13). As noticed in [59], the absence of flat directions in the $\frac{1}{2}$-BPS attractor flow (which is a general feature of $\mathcal{N}=2, d=4$ ungauged supergravity 
coupled to Abelian vector multiplets, at least as far as the metric of the scalar manifold is strictly positive definite $\forall \tau \in \mathbb{R}^{-}$[10]) is crucial for the validity of the expression (9). As pointed out in Sect. 4., the same holds for the non-BPS $Z=0$ case. Indeed, a consequence of the general form of non-BPS $Z=0$ attractor flow given by Eq. (29) is that $\Gamma_{\infty}$ satisfies the non-BPS $Z=0$ Attractor Eqs. (see e.g. [23] and [32]), determining a sort of "Attractor Mechanism at spatial infinity". Analogously to what happens in the $\frac{1}{2}$-BPS case, the absence of flat directions in the non-BPS $Z=0$ attractor flow (which is not a general feature of $\mathcal{N}=2, d=4$ ungauged supergravity coupled to Abelian vector multiplets, but however holds for the stu model [40, 43]) is crucial for the validity of the expression (29). In view of the crucial differences among the non-BPS $Z \neq 0$ attractor flow and the $\frac{1}{2}$-BPS and non-BPS $Z=0$ attractor flows (such as the presence of a 2-dim. real moduli space $(S O(1,1))^{2}$ all along the non-BPS $Z \neq 0$ attractor flow), it would be interesting to investigate the non-BPS $Z \neq 0$ "Attractor Mechanism at spatial infinity", if any.

\section{Acknowledgements}

We would like to warmly acknowledge L. Andrianopoli, A. Ceresole, G. Dall'Agata, R. D'Auria, E. Orazi, M. Trigiante, and especially E. G. Gimon, for fruitful discussions and interest in this work. We also thank A. Banijamali for careful reading of our manuscript and correcting some typos.

A. M. would also like to thank the Department of Physics, Theory Unit Group at CERN, where part of this work was done, for kind hospitality and stimulating environment.

The work of S.B. has been supported in part by the European Community Human Potential Program under contract MRTN-CT-2004-005104 "Constituents, fundamental forces and symmetries of the Universe".

The work of S.F. has been supported in part by the European Community Human Potential Program under contract MRTN-CT-2004-005104 "Constituents, fundamental forces and symmetries of the Universe”, in association with INFN Frascati National Laboratories and by D.O.E. grant DE-FG0391ER40662, Task C.

The work of A.M. has been supported by Museo Storico della Fisica e Centro Studi e Ricerche "Enrico Fermi", Rome, Italy, in association with INFN-LNF, and by an INFN Visiting Theoretical Fellowship at Stanford Institute for Theoretical Physics.

The work of A.Y. was supported in part by the grant INTAS-05-7928, in association with INFN Frascati National Laboratories.

\section{References and Notes}

1. Black Holes; De Witt, B.; De Witt C., Eds.; Gordon and Breach: New York, 1973. Hawking, S. W.; Israel, W. General Relativity; Cambridge University Press: Cambridge, 1979. Wald, R. M. General Relativity; University of Chicago Press: Chicago, 1984.

2. Moore, G. W. Les Houches lectures on strings and arithmetic, arXiv: hepth/0401049. Douglas, M. R.; Reinbacher, R.; Yau, S. T. Branes, bundles and attractors: Bogomolov and beyond, arXiv:math.ag/0604597.

3. Duff, M. J. String Triality, Black Hole Entropy and Cayley's Hyperdeterminant. Phys. Rev. D 2007, 76, 025017-025026, hep-th/0601134. Kallosh, R.; Linde, A. Strings, black holes, and 
quantum information. Phys. Rev. D 2006, 73, 104033-104060, hep-th/0602061. Levay, P. Stringy black holes and the geometry of entanglement. Phys. Rev. D 2006, 74, 024030-024065, hep-th/0603136. Duff, M. J.; Ferrara, S. $E_{7}$ and the tripartite entanglement of seven qubits. Phys. Rev. D 2007, 76, 025018-025032, quant-ph/ 0609227 . Levay, P. Strings, black holes, the tripartite entanglement of seven qubits and the Fano plane. Phys. Rev. D 2004, 75, 024024024067, hep-th/ 0610314 . Duff, M. J.; Ferrara, S. Black hole entropy and quantum information. hepth/0612036. Levay, P. A Three-qubit interpretation of BPS and non-BPS STU black holes. Phys. Rev. D 2007, 76, 106011-106046, arXiv:0708.2799. Borsten, L.; Dahanayake, D.; Duff, M. J.; Rubens, W.; Ebrahim, H. Wrapped branes as qubits. Phys. Rev. Lett. 2008, 100, 251602-251606, arXiv:0802.0840.

4. Hawking, S. W.; Penrose, R. The singularities of gravitational collapse and cosmology. Proc. Roy. Soc. Lond. A 1970, 314, 529-548.

5. Penrose, R. Gravitational collapse: the role of general relativity. Riv. Nuovo Cim. 1969, 1, 252276. Gen. Rel. Grav. 2002, 34, 1141-1165 (special number). Penrose, R. In: General Relativity, an Einstein Centenary Survey, Hawking, S. W.; Israel, W., Eds.; Cambridge University Press: Cambridge, 1979.

6. Gibbons, G. W. In: Unified theories of Elementary Particles. Critical Assessment and Prospects. Proceedings of the Heisemberg Symposium, Munchen, Germany, 1981, Breitenlohner, P; Durr, H. P., Eds., Lecture Notes in Physics 160, Springer-Verlag: Berlin, 1982. Gibbons, G. W. In: Supersymmetry, Supergravity and Related Topics. Proceedings of the XVth GIFT International Physics, Girona, Spain, 1984, del Aguila, F.; de Azcárraga, J.; Ibáñez, L., Eds., World Scientific: Singapore, 1985, p. 147. Breitenlohner, P.; Maison, D.; Gibbons, G. W. 4-dimensional black holes from Kaluza-Klein theories. Commun. Math. Phys. 1988, 120, 295-347. Kallosh, R. Supersymmetric black holes. Phys. Lett. B 1992, 282, 80-88, hep-th/9201029. Khuri, R. R.; Ortín, T. Supersymmetric black holes in $\mathcal{N}=8$ supergravity. Nucl. Phys. B 1996, 467, 355382, hep-th/9512177. Sen, A. Black-Hole Solutions in Heterotic String Theory on a Torus. Nucl. Phys. B 1995, 440, 421-440, hep-th/9411187. Sen, A. Quantization of dyon charge and electric-magnetic duality in string theory. Phys. Lett. B 1993, 303, 22-26, hep-th/9209016. Sen, A. Extremal Black-Holes and Elementary String States. Mod. Phys. Lett. A 1995, 10, 2081-2094, hep-th/9504147. Cvetic, M.; Hull, C. M. Black holes and U-duality. Nucl. Phys. B 1996, 480, 296-316, hep-th/9606193. Cvetic, M.; Gaida, I. Duality-invariant nonextreme black holes in toroidally compactified string theory. Nucl. Phys. B 1997, 505, 291-306, hep-th/9703134. Cvetic, M.; Youm, D. All the Static Spherically Symmetric Black Holes of Heterotic String on a Six Torus. Nucl. Phys. B 1996, 472, 249-267, hepth/9512127. Cvetic, M. ; Tseytlin, A. A. Solitonic strings and BPS saturated dyonic black holes. Phys. Rev. D 1996, 53, 5619-5633 [Erratum-ibid. 1997, 55, 3907], hep-th/9512031.

7. Ferrara, S.; Kallosh, R.; Strominger, A. $\mathcal{N}=2$ extremal black holes. Phys. Rev. D 1995, 52, 5412-5416, hep-th/9508072.

8. Ferrara, S.; Kallosh, R. Supersymmetry and attractors. Phys. Rev. D 1996, 54, 1514-1524, hep-th/9602136. Ferrara, S.; Kallosh, R. Universality of supersymmetric attractors. Phys. Rev. D 1996, 54, 1525-1534, hep-th/ 9603090. 
9. Strominger, A. Macroscopic entropy of $\mathcal{N}=2$ extremal black holes. Phys. Lett. B 1996, 383, 39-43, hep-th/9602111.

10. Ferrara, S. ; Gibbons, G. W.; Kallosh, R. Black Holes and Critical Points in Moduli Space. Nucl. Phys. B 1997, 500, 75-93, hep-th/9702103.

11. Sen, A. Black Hole Entropy Function and the Attractor Mechanism in Higher Derivative Gravity. JHEP 2005, 09, 038-050, hep-th/ 0506177.

12. Goldstein, K.; Iizuka, N.; Jena, R. P.; Trivedi, S. P. Non-Supersymmetric Attractors. Phys. Rev. D 2005, 72, 124021-124073, hep-th/0507096.

13. Sen, A. Entropy Function for Heterotic Black Holes. JHEP 2006, 03, 008-030, hep-th / 0508042.

14. Kallosh, R. New Attractors. JHEP 2005, 0512, 022-029, hep-th/ 0510024.

15. Tripathy, P. K.; Trivedi, S. P. Non-Supersymmetric Attractors in String Theory. JHEP 2006, 0603, 022-054, hep-th/0511117.

16. Giryavets, A. New Attractors and Area Codes. JHEP 2006, 0603, 020-036, hep-th / 0511215.

17. Goldstein, K.; Jena, R. P.; Mandal, G.; Trivedi, S. P. A C-Function for Non-Supersymmetric Attractors. JHEP 2006, 0602, 053-075, hep-th/ 0512138.

18. Alishahiha, M.; Ebrahim, H. Non-supersymmetric attractors and entropy function. JHEP 2006, 0603, 003-018, hep-th/0601016.

19. Kallosh, R.; Sivanandam, N.; Soroush, M. The Non-BPS Black Hole Attractor Equation. JHEP 2006, 0603, 060-092, hep-th/ 0602005.

20. Chandrasekhar, B.; Parvizi, S.; Tavanfar, A.; Yavartanoo, H. Non-supersymmetric attractors in $R^{2}$ gravities. JHEP 2006, 0608, 004-023, hep-th/ 0602022.

21. Hsu, J. P.; Maloney, A.; Tomasiello, A. Black Hole Attractors and Pure Spinors. JHEP 2006, 0609, 048-074, hep-th/0602142.

22. Bellucci, S.; Ferrara, S.; Marrani, A. On some properties of the Attractor Equations. Phys. Lett. B 2006, 635, 172-179, hep-th/ 0602161.

23. Bellucci, S.; Ferrara, S.; Marrani, A. Supersymmetric Mechanics. Vol.2: The Attractor Mechanism and Space-Time Singularities, LNP 701; Springer-Verlag: Heidelberg, 2006.

24. Ferrara, S.; Kallosh, R. On $\mathcal{N}=8$ attractors. Phys. Rev. D 2006, 73, 125005-125020, hep-th/0603247.

25. Alishahiha, M.; Ebrahim, H. New attractor, Entropy Function and Black Hole Partition Function. JHEP 2006, 0611, 017-041, hep-th/ 0605279.

26. Bellucci, S.; Ferrara, S.; Günaydin, M.; Marrani, A. Charge Orbits of Symmetric Special Geometries and Attractors. Int. J. Mod. Phys. A 2006, 21, 5043-5098, hep-th / 0606209.

27. Astefanesei, D.; Goldstein, K.; Jena, R. P.; Sen, A.; Trivedi, S. P. Rotating Attractors. JHEP 2006, 0610, 058-105, hep-th/0606244.

28. Kallosh, R.; Sivanandam, N.; Soroush, M. Exact Attractive non-BPS STU Black Holes. Phys. Rev. D 2006, 74, 065008-065032, hep-th/ 0606263.

29. Kaura, P.; Misra, A. On the Existence of Non-Supersymmetric Black Hole Attractors for TwoParameter Calabi-Yau's and Attractor Equations. Fortsch. Phys. 2006, 54, 1109-1141, hep-th/0607132.

30. Cardoso, G. L.; Grass, V.; Lüst, D.; Perz, J. Extremal non-BPS Black Holes and Entropy Extrem- 
ization. JHEP 2006, 0609, 078-097, hep-th/ 0607202.

31. Morales, J. F.; Samtleben, H. Entropy function and attractors for AdS black holes. JHEP 2006, 0610, 074-101, hep-th/0608044.

32. Bellucci, S.; Ferrara, S.; Marrani, A.; Yeranyan, A. Mirror Fermat Calabi-Yau Threefolds and Landau-Ginzburg Black Hole Attractors. Riv. Nuovo Cim. 2006, 029, 1-88, hep-th/ 0608091.

33. Astefanesei, D.; Goldstein, K.; Mahapatra, S. Moduli and (un)attractor black hole thermodynamics. Gen. Rel. Grav. Online First, hep-th/0611140.

34. Cardoso, G.L.; de Wit, B.; Mahapatra, S. Black hole entropy functions and attractor equations. JHEP 2007, 0703, 085-107, hep-th/ 0612225.

35. D'Auria, R.; Ferrara, S.; Trigiante, M. Critical points of the Black-Hole potential for homogeneous special geometries. JHEP 2007, 0703, 097-114, hep-th/ 0701090.

36. Bellucci, S.; Ferrara, S. Marrani, A. Attractor Horizon Geometries of Extremal Black Holes. Contribution to the Proceedings of the XVII SIGRAV Conference,4-7 September 2006, Turin, Italy, hep-th/0702019.

37. Ceresole, A.; Dall'Agata, G. Flow Equations for Non-BPS Extremal Black Holes. JHEP 2007, 0703, 110-131, hep-th/0702088.

38. Andrianopoli, L.; D'Auria, R.; Ferrara, S.; Trigiante, M. Black Hole Attractors in $\mathcal{N}=1$ Supergravity. JHEP 2007, 0707, 019-042, hep-th / 0703178.

39. Saraikin, K.; Vafa, C. Non-supersymmetric Black Holes and Topological Strings. Class. Quant. Grav. 2008, 25, 095007-095054, hep-th/ 0703214.

40. Ferrara, S.; Marrani, A. $\mathcal{N}=8$ non-BPS Attractors, Fixed Scalars and Magic Supergravities. Nucl. Phys. B 2008, 788, 63-88, arXiv:0 005.3866.

41. Nampuri, S.; Tripathy, P. K.; Trivedi, S. P. On The Stability of Non-Supersymmetric Attractors in String Theory. JHEP 2007, 0708, 054-074, arXiV: 0705 . 4554.

42. Andrianopoli, L.; D'Auria, R.; Orazi, E.; Trigiante, M. First Order Description of Black Holes in Moduli Space. JHEP 2007, 0711, 032-060, arXiV: 0706.0712.

43. Ferrara, S.; Marrani, A. On the Moduli Space of non-BPS Attractors for $\mathcal{N}=2$ Symmetric Manifolds. Phys. Lett. B 2007, 652, 111-117, arXiV:0706.1667.

44. Astefanesei, D.; Yavartanoo, H. Stationary black holes and attractor mechanism. Nucl. Phys. B 2008, 794, 13-27, arXiv:0 706.1847.

45. Cardoso, G. L.; Ceresole, A.; Dall'Agata, G.; Oberreuter, J. M.; Perz, J. First-order flow equations for extremal black holes in very special geometry. JHEP 2007, 0710, 063-084, arXiV:0706.3373.

46. Misra, A.; Shukla, P. Moduli stabilization, large-volume dS minimum without D3-bar branes, (non)supersymmetric black hole attractors and two-parameter Swiss cheese Calabi-Yau's. Nucl. Phys. B 2008, 799, 165-198, arXiV: 0707.0105.

47. Ceresole, A.; Ferrara, S.; Marrani, A. 4d/5d Correspondence for the Black Hole Potential and its Critical Points. Class. Quant. Grav. 2007, 24, 5651-5666, arXiV: 0707.0964.

48. Anber, M. M.; Kastor, D. The Attractor mechanism in Gauss-Bonnet gravity. JHEP 2007, 0710, 084-100, arXiv:0707.1464.

49. Myung, Y. S.; Kim, Y.-W.; Park, Y.-J. New attractor mechanism for spherically symmetric extremal 
black holes. Phys. Rev. D 2007, 76, 104045-104064, arXiv: 0707.1933.

50. Bellucci, S.; Marrani, A.; Orazi, E.; Shcherbakov, A. Attractors with Vanishing Central Charge. Phys. Lett. B 2007, 655, 185-195, arXiV: 0707.2730.

51. Hotta, K.; Kubota, T. Exact Solutions and the Attractor Mechanism in Non-BPS Black Holes. Prog. Theor. Phys. 2007, 118, 969-981, arXiv:0707. 4554.

52. Gao, X. Non-supersymmetric Attractors in Born-Infeld Black Holes with a Cosmological Constant. JHEP 2007, 0711, 006-024, arXiv: 0708 . 1226.

53. Ferrara, S.; Marrani, A. Black Hole Attractors in Extended Supergravity. Contribution to the Proceedings of 13th International Symposium on Particles, Strings and Cosmology (PASCOS 07), London, England, 2-7 July 2007. AIP Conf. Proc. 2007, 957, 58-68, arXiv : 0708 . 1268.

54. Sen, A. Black Hole Entropy Function, Attractors and Precision Counting of Microstates. arXiv:0708.1270.

55. Belhaj, A.; Drissi, L. B.; Saidi, E. H.; Segui, A. $\mathcal{N}=2$ Supersymmetric Black Attractors in Six and Seven Dimensions. Nucl. Phys. B 2008, 796, 521-580 (2008), arXiv: 0709 . 0398.

56. Andrianopoli, L.; Ferrara, S.; Marrani, A.; Trigiante, M. Non-BPS Attractors in $5 \mathrm{~d}$ and $6 \mathrm{~d}$ Extended Supergravity. Nucl. Phys. B 2008, 795, 428-452, arXiv: 0709.3488.

57. Gaiotto, D.; Li, W.; Padi, M. Non-Supersymmetric Attractor Flow in Symmetric Spaces. JHEP 2007, 0712, 093-145, arXiv:0710.1638.

58. Bellucci, S.; Ferrara, S.; Marrani, A.; Shcherbakov, A. Splitting of Attractors in 1-modulus Quantum Corrected Special Geometry. JHEP 2008, 0802, 088-101, arXiv: 0710 . 3559.

59. Gimon, E. G.; Larsen, F.; Simon, J. Black Holes in Supergravity: the non-BPS Branch. JHEP 2008, 0801, 040-070, arXiv:0710.4967.

60. Astefanesei, D.; Nastase, H.; Yavartanoo, H.; Yun, S. Moduli flow and non-supersymmetric AdS attractors. JHEP 2008, 0804, 074-115, arXiv: 0711.0036.

61. Bellucci, S.; Ferrara, S.; Kallosh, R.; Marrani, A. Extremal Black Hole and Flux Vacua Attractors. Contribution to the Proceedings of the Winter School on Attractor Mechanism 2006 (SAM2006), 20-24 March 2006, INFN-LNF, Frascati, Italy, arXiv: 0711.4547.

62. Cai, R.-G.; Pang, D.-W. A Note on exact solutions and attractor mechanism for non-BPS black holes. JHEP 2008, 0801, 046-061, arXiv: 0712 . 0217.

63. Huebscher, M.; Meessen, P.; Ortín, T.; Vaulà, S. Supersymmetric $\mathcal{N}=2$ Einstein-Yang-Mills monopoles and covariant attractors. arXiv:0712.1530.

64. Li, W. Non-Supersymmetric Attractors in Symmetric Coset Spaces. Contribution to the Proceedings of 3rd School on Attractor Mechanism (SAM 2007), Frascati, Italy, 18-22 Jun 2007, arXiv:0801.2536.

65. Bellucci, S.; Ferrara, S.; Marrani, A.; Yeranyan, A. $d=4$ Black Hole Attractors in $\mathcal{N}=2$ Supergravity with Fayet-Iliopoulos Terms. Phys. Rev. D 2008, 77, 085027-085061, arXiv: 0802.0141.

66. Saidi, E. H. BPS and non BPS 7D Black Attractors in $M$-Theory on K3. arXiv: 0802.0583.

67. Saidi, E. H. On Black Hole Effective Potential in $6 D / 7 D \mathcal{N}=2$ Supergravity. arXiv: 0803.0827.

68. Saidi, E. H.; Segui, A. Entropy of Pairs of Dual Attractors in six and seven Dimensions. arXiv:0803.2945.

69. Bellucci, S.; Ferrara, S.; Marrani, A. Attractors in Black. Contribution to the Proceedings of the 3rd 
RTN Workshop "Constituents, Fundamental Forces and Symmetries of the Universe", 1-5 October 2007, Valencia, Spain, Fortsch. Phys. 2008, 56,761-785, arXiv: 0805.1310.

70. Ferrara, S.; Hayakawa, K.; Marrani, A. Erice Lectures on Black Holes and Attractors. Contribution to the Proceedings of the International School of Subnuclear Physics: 45th Course: Searching for the "Totally Unexpected" in the LHC Era, Erice, Sicily, Italy, 29 Aug - 7 Sep 2007, arXiv:0805.2498.

71. Astefanesei, D.; Banerjee, N.; Dutta, S. (Un)attractor black holes in higher derivative AdS gravity. arXiv:0806.1334.

72. Huebscher, M.; Meessen, P.; Ortín, T. Vaulà, S. $\mathcal{N}=2$ Einstein-Yang-Mills's BPS solutions. arXiv:0806.1477.

73. Bergshoeff, E.; Chemissany, W.; Ploegh, A.; Trigiante, M.; Van Riet, T. Generating Geodesic Flows and Supergravity Solutions. arXiv:0806.2310.

74. Ferrara, S.; Gnecchi, A.; Marrani, A. $d=4$ Attractors, Effective Horizon Radius and Fake Supergravity. arXiv:0806.3196.

75. Ooguri, H.; Strominger, A.; Vafa, C. Black Hole Attractors and the Topological String. Phys. Rev. D 2004, 70, 106007-106039, hep-th/ 0405146.

76. Ooguri, H.; Vafa, C.; Verlinde, E. Hartle-Hawking wave-function for flux compactifications: the Entropic Principle. Lett. Math. Phys. 2005, 74, 311-342, hep-th/ 0502211.

77. Aganagic, M.; Neitzke, A.; Vafa, C. BPS microstates and the open topological string wave function. hep-th/0504054.

78. Gukov, S.; Saraikin, K.; Vafa, C. The Entropic Principle and Asymptotic Freedom. Phys. Rev. D 2006, 73, 066010-066035, hep-th/0509109.

79. Van Nieuwenhuizen, P. Supergravity. Phys. Rept. 1981, 68, 189-398.

80. For reviews on black holes in superstring theory see e.g.: Maldacena, J. M. Black-Holes in String Theory. hep-th/9607235. Peet, A. W. TASI lectures on black holes in string theory. arXiv: hep-th/0008241. Pioline, B. Lectures on black holes, topological strings and quantum attractors. Class. Quant. Grav. 2006, 23, S981-S107, hep-th/0607227. Dabholkar, A. Black hole entropy and attractors. Class. Quant. Grav. 2006, 23, S957-S980.

81. For recent reviews, see e.g.: Schwarz, J. H. Lectures on superstring and $M$-theory dualities. Nucl. Phys. Proc. Suppl. 1997, 55, 1-32, hep-th/9607201. Duff, M. J. M-theory (the theory formerly known as strings). Int. J. Mod. Phys. A 1996, 11, 5623-5642, hep-th/9608117. Sen, A. Unification of string dualities. Nucl. Phys. Proc. Suppl. 1997, 58, 5-19, hep-th/ 9609176.

82. Schwarz, J. H.; Sen, A. Duality symmetries of $4 D$ heterotic strings. Phys. Lett. B 1993, 312, 105-114, hep-th/9305185. Schwarz, J. H.; Sen, A. Duality Symmetrical Actions. Nucl. Phys. B 1994, 411, 35-63, hep-th/ 9304154.

83. Gasperini, M.; Maharana, J.; Veneziano, G. From trivial to non-trivial conformal string backgrounds via $O(d, d)$ transformations. Phys. Lett. B 1991, 272, 277-284. Maharana, J.; Schwarz, J. H. Noncompact Symmetries in String Theory. Nucl. Phys. B 1993, 390, 3-32, hep-th/ 9207016.

84. Witten, E. String Theory Dynamics in Various Dimensions. Nucl. Phys. B 1995, 443, 85-126, hep-th/9503124.

85. Schwarz, J. H. $M$-theory extensions of $T$ duality. arXiv: hep-th/9601077. Vafa, C. Evidence 
for F-theory. Nucl. Phys. B 1996, 469, 403-418, hep-th/ 9602022.

86. Becker, K.; Becker, M.; Schwarz, J. H. String theory and M-theory: A modern introduction; Cambridge University Press: Cambridge, 2007.

87. Nordström, G. On the energy of gravitational field in Einstein's theory. Proc. Kon. Ned. Akad. Wet. 1918, 20, 1238. Reissner, H. Uber die Eigengravitation des elektrishen Feldes nach der Einsteinschen Theorie. Ann. Physik 1916, 50, 106.

88. Smarr, L. Mass Formula for Kerr Black Hole. Phys. Rev. Lett. 1973, 30, 71-73.

89. Arnowitt, R.; Deser, S.; Misner, C. W. Canonical Variables for General Relativity. Phys. Rev. 1960, 117, 1595-16025.

90. Deser, S.; Teitelboim, C. Supergravity Has Positive Energy. Phys. Rev. Lett. 1977, 39, 249-258.

91. Bondi, H.; van der Burg, M. G. J.; Metzner, A. W. K. Gravitational waves in general relativity. 7. Waves from axisymmetric isolated systems. Proc. Roy. Soc. Lond. A 1962, 269, 21-52.

92. Kallosh, R.; Ortín, T. Charge quantization of axion-dilaton black holes. Phys. Rev. D 1993, 48, 742-747, hep-th/9302109. Bergshoeff, E.; Kallosh, R.; Ortín, T. Stationary axion/dilaton solutions and supersymmetry. Nucl. Phys. B 1996, 478, 156-180, hep-th/9605059.

93. Kallosh, R.; Linde, A. D.; Ortín, T; Peet, A. W.; Van Proeyen, A. Supersymmetry as a cosmic censor. Phys. Rev. D 1992, 46, 5278-5302, hep-th/9205027.

94. Gibbons, G. W.; Kallosh, R.; Kol, B. Moduli, Scalar Charges, and the First Law of Black Hole Thermodynamics. Phys. Rev. Lett. 1996, 77, 4992-4995, hep-th/9607108.

95. Hawking, S. W. Gravitational Radiation from Colliding Black Holes. Phys. Rev. Lett. 1971, 26, 1344-1346. Bekenstein, J. D. Black Holes and Entropy. Phys. Rev. D 1973, 7, 2333-2346.

96. Bertotti, B. Uniform Electromagnetic Field in the Theory of General Relativity. Phys. Rev. 1959, 116, 1331. Robinson, I. A solution of the Maxwell-Einstein equations. Bull. Acad. Pol. Sci. Ser. Sci. Math. Astron. Phys. 1959, 7, 351-352.

97. Cremmer, E.; Kounnas, C.; Van Proeyen, A.; Derendinger, J. P.; Ferrara, S.; de Wit, B.; Girardello, L. Vector Multiplets Coupled to $\mathcal{N}=2$ Supergravity: SuperHiggs Effect, Flat Potentials and Geometric Structure. Nucl. Phys. B 1985, 250, 385-444.

98. Duff, M. J.; Liu, J. T.; Rahmfeld, J. Four-dimensional string/string/string triality. Nucl. Phys. B 1996, 459, 125-159, hep-th/9508094.

99. Behrndt, K.; Kallosh, R.; Rahmfeld, J.; Shmakova, M.; Wong, W. K. STU Black Holes and String Triality. Phys. Rev. D 1996, 54, 6293-6301, hep-th/9608059.

100. Shmakova, M. Calabi-Yau black holes. Phys. Rev. D 1997, 56, 540-544, hep-th/ 9612076.

101. Hull, C.; Townsend, P. K. Unity of Superstring Dualities. Nucl. Phys. B 1995, 438, 109-137, hep-th/9410167.

102. Tseytlin, A. A. Harmonic superpositions of $M$-branes. Nucl. Phys. B 1996, 475, 149-163, hep-th/9604035.

103. Gauntlett, J. P.; Kastor, D. A.; Traschen, J. H. Overlapping branes in $M$ theory. Nucl. Phys. B 1996, 478, 544-560, hep-th/ 9604179.

104. Balasubramanian, V.; Larsen, F. On D-branes and black holes in four-dimensions. Nucl. Phys. B 1996, 478, 199-208, hep-th/9604189.

105. Cvetic, M.; Youm, D. Dyonic BPS saturated black holes of heterotic string on a six torus. Phys. 
Rev. D 1996, 53, 584-588, hep-th/ 9507090.

106. Behrndt, K.; Lüst, D.; Sabra, W. A. Stationary solutions of $\mathcal{N}=2$ supergravity. Nucl. Phys. $B$ 1998, 510, 264-288, hep-th/9705169.

107. Sabra, W. A. Black holes in $\mathcal{N}=2$ supergravity theories and harmonic functions. Nucl. Phys. $B$ 1998, 510, 247-263, hep-th/9704147.

108. Sabra, W. A. General static $\mathcal{N}=2$ black holes. Mod. Phys. Lett. A 1997, 12, 2585-2590, hep-th/9703101.

109. Balasubramanian, V.; Gimon, E. G.; Levi, T. S. Four Dimensional Black Hole Microstates: From D-branes to Spacetime Foam. JHEP 2008, 0801, 056-088, hep-th / 0606118.

110. Bates, B.; Denef, F. Exact solutions for supersymmetric stationary black hole composites. hep-th/0304094.

111. Rahmfeld, J. Extremal black holes as bound states. Phys. Lett. B 1996, 372, 198-203, hep-th/9512089. Duff, M. J.; Rahmfeld, J. Bound states of black holes and other $p$-branes. Nucl. Phys. B 1996, 481, 332-352, hep-th/9605085. Denef, F. Supergravity flows and Dbrane stability. JHEP 2000, 0008, 050-091, hep-th/0 00504 9. Gaiotto, D.; Simons, A.; Strominger, A.; Yin, X. D0-branes in black hole attractors. hep-th/0412179. Ritz, A.; Shifman, M. A.; Vainshtein, A. I.; Voloshin, M. B. Marginal stability and the metamorphosis of BPS states. Phys. Rev. D 2001, 63, 065018-065071, hep-th/0006028. Aspinwall, P. S.; Maloney, A.; Simons, A. Black hole entropy, marginal stability and mirror symmetry. JHEP 2007, 0707, 034-059, hep-th/0610033. Sen, A. Walls of Marginal Stability and Dyon Spectrum in $\mathcal{N}=4$ Supersymmetric String Theories. JHEP 2007, 0705, 039-081, hep-th/ 0702141. Denef, F.; Gaiotto, D.; Strominger, A.; Van den Bleeken, D.; Yin, X. Black Hole Deconstruction. hep-th/0703252. Sen, A. $\mathcal{N}=8$ Dyon Partition Function and Walls of Marginal Stability. JHEP 2008, 0807, 118-140, arXiv:0803.1014.

112. Gibbons, G. W.; Hull, C. H. A Bogomol'ny bound for general relativity and solitons in $\mathcal{N}=2$ supergravity. Phys. Lett. B 1982, 109, 190-204.

113. Cecotti, S.; Ferrara, S.; Girardello, L. Geometry of Type II Superstrings and the Moduli of Superconformal Field Theories. Int. J. Mod. Phys. A 1989, 4, 2475-2558.

114. Günaydin, M.; Sierra, G.; Townsend, P. K. The Geometry of $\mathcal{N}=2$ Maxwell-Einstein Supergravity and Jordan Algebras. Nucl. Phys. B 1984, 242, 244-291.

115. Cremmer, E.; Van Proeyen, A. Classification of Kähler Manifolds in $\mathcal{N}=2$ Vector Multiplet Supergravity Couplings. Class. Quant. Grav. 1985, 2, 445-462.

116. de Wit, B.; Vanderseypen, F.; Van Proeyen, A. Symmetry Structures of Special Geometries. Nucl. Phys. B 1993, 400, 463-524, hep-th/9210068.

117. de Wit, B.; Van Proeyen, A. Special geometry, cubic polynomials and homogeneous quaternionic spaces. Commun. Math. Phys. 1992, 149, 307-334, hep-th/9112027.

118. Freedman, D. Z.; Nunez, C.; Schnabl, M.; Skenderis, K. Fake supergravity and domain wall stability. Phys. Rev. D 2004, 69, 104027-104063, hep-th/0312055. Celi, A.; Ceresole, A.; Dall'Agata, G.; Van Proeyen, A.; Zagermann, M. On the fakeness of fake supergravity. Phys. Rev. D 2005, 71, 045009-045045, hep-th/ 0410126 . Zagermann, M. $\mathcal{N}=4$ fake supergravity. Phys. Rev. D 2005, 71, 125007-125030, hep-th/0412081. Skenderis, K.; Townsend, P. K. 
Hidden supersymmetry of domain walls and cosmologies. Phys. Rev. Lett. 2006, 96, 19130119306, hep-th/ 0602260 . Bazeia, D.; Gomes, C.B.; Losano, L.; Menezes, R. First-order formalism and dark energy. Phys. Lett. B 2006, 633, 415-419, astro-ph/0512197. Skenderis, K.; Townsend, P. K. Pseudo-Supersymmetry and the Domain-Wall/Cosmology Correspondence. $J$. Phys. A 2007, 40, 6733-6742, hep-th/ 0610253.

119. Ceresole, A.; D'Auria, R.; Ferrara, S. The Symplectic Structure of $\mathcal{N}=2$ Supergravity and Its Central Extension. Talk given at ICTP Trieste Conference on Physical and Mathematical Implications of Mirror Symmetry in String Theory, Trieste, Italy, 5-9 June 1995. Nucl. Phys. Proc. Suppl. 1996, 46, 67-74, hep-th/9509160.

120. Levay, P.; Saniga, M.;Vrana, P. Three-Qubit Operators, the Split Cayley Hexagon of Order Two and Black Holes. arXiv:0808.3849.

121. Ferrara, S.; Gimon, E. G.; Kallosh, R. Magic supergravities, $\mathcal{N}=8$ and black hole composites. Phys. Rev. D 2006, 74, 125018-125053, hep-th/0606211.

122. Gilmore, R. Lie Groups, Lie Algebras, and Some of Their Applications; Dover Publications: New York, 2006.

123. Klich, I.; Levitov, L. Quantum Noise as an Entanglement Meter. arXiv:0804.1377.

(c) 2008 by the authors; licensee Molecular Diversity Preservation International, Basel, Switzerland. This article is an open-access article distributed under the terms and conditions of the Creative Commons Attribution license (http://creativecommons.org/licenses/by/3.0/). 